\title{
Landscape of promising nonsupersymmetric string models
}

\author{
Ricardo Pérez-Martínez@ ${ }^{1, *}$ Saúl Ramos-Sánchez@ ${ }^{2, \dagger}$ and Patrick K. S. Vaudrevange $\circledast^{3, \$}$ \\ ${ }^{1}$ Facultad de Ciencias Físico-Matemáticas, Universidad Autónoma de Coahuila, \\ Edificio A, Unidad Camporredondo, 25000, Saltillo, Coahuila, México \\ ${ }^{2}$ Instituto de Física, Universidad Nacional Autónoma de México, POB 20-364, Cd.Mx. 01000, México \\ ${ }^{3}$ Physik Department T75, Technische Universität München, \\ James-Franck-Straße 1, 85748 Garching, Germany
}

(Received 16 May 2021; accepted 26 July 2021; published 30 August 2021)

\begin{abstract}
Leptoquarks extending the Standard Model (SM) have attracted increased attention in the recent literature. Hence, the identification of four-dimensional (4D) SM-like models and the classification of allowed leptoquarks from strings is an important step in the study of string phenomenology. We perform the most extensive search for SM-like models from the nonsupersymmetric heterotic string $\mathrm{SO}(16) \times \mathrm{SO}(16)$, resulting in more than 170000 inequivalent promising string models from 138 Abelian toroidal orbifolds. We explore the 4D massless particle spectra of these models in order to identify all exotics beside the three generations of quarks and leptons. Hereby, we learn which leptoquark can be realized in this string setup. Moreover, we analyze the number of SM Higgs doublets which is generically larger than one. Then, we identify SM-like models with a minimal particle content. These so-called almost SM models appear most frequently in the orbifold geometries $\mathbb{Z}_{2} \times \mathbb{Z}_{4}(2,4)$ and $(1,6)$. Finally, we apply machine learning to our data set in order to predict the orbifold geometry where a given particle spectrum is most likely to be found.
\end{abstract}

DOI: $10.1103 /$ PhysRevD.104.046026

\section{INTRODUCTION}

Compactifying six of the ten dimensions of string theory is a crucial step towards four-dimensional (4D) string phenomenology. Most of the studies in this context have been performed in theories with space-time supersymmetry (SUSY). In particular, toroidal orbifold compactifications of the heterotic string [1-3] have been shown to yield a large amount of semirealistic models [4-16] with which different phenomenological questions can be addressed [17-26]. However, since SUSY has not been detected yet, it is worthwhile to entertain the possibility that nature might be described by a non-SUSY model emerging directly from string theory. In this sense, the non-SUSY tachyon-free heterotic string with the $\mathrm{SO}(16) \times \mathrm{SO}(16)$ gauge group in ten dimensions [27-29] can play a special role. In order to compare to supersymmetric string models of particle physics, a natural question is whether appealing phenomenology can also arise from non-SUSY string theory compactified on SUSY-preserving spaces.

\footnotetext{
*ricardo.perezmartinez@uadec.edu.mx

ramos@fisica.unam.mx

"patrick.vaudrevange@tum.de
}

Published by the American Physical Society under the terms of the Creative Commons Attribution 4.0 International license. Further distribution of this work must maintain attribution to the author(s) and the published article's title, journal citation, and DOI. Funded by SCOAP.
One can define an orbifold as the quotient of a sixdimensional (6D) torus over a discrete set of its isometries, among which the rotational isometries build the so-called point group. There are 7103 admissible point groups in six dimensions. 52 of them can leave $\mathcal{N}=1$ supersymmetry unbroken in 4D [30], out of which 17 are Abelian $\left(\mathbb{Z}_{N}\right.$ and $\mathbb{Z}_{N} \times \mathbb{Z}_{M}$ for various orders $M$ and $N$ ). These 17 point groups give rise to in total 138 inequivalent orbifolds. These orbifolds have been the starting point for many studies trying to connect string theory to the supersymmetric extension of the Standard Model (SM). In order to contrast SUSY and non-SUSY phenomenology, in this work we focus on orbifold compactifications of the nonSUSY $\mathrm{SO}(16) \times \mathrm{SO}(16)$ heterotic string based on these 138 orbifold geometries.

The quest to connect heterotic string theory with nonSUSY particle phenomenology is not new. Models with promising properties have been constructed using orbifolds in the bosonic [31] and fermionic formulations [32-35], Calabi-Yau manifolds [36], and so-called coordinatedependent compactifications $[37,38]$. Also, string models with spontaneously broken SUSY have been considered [39-41]. However, our present study represents the most extensive search to date for non-SUSY string models that reproduce features of the SM of particle physics.

By using the orbifolder program [42], modified to the construction of string models without SUSY, we obtain more than 170000 inequivalent SM-like models from the 
TABLE I. Total number of inequivalent SM-like string models obtained in our extensive search using 138 orbifold geometries classified in Ref. [30]. We find 170219 inequivalent SM-like models in 104 orbifold geometries. In the "orbifold" columns we label the considered orbifold geometries by their point group $\left(\mathbb{Z}_{N}\right.$ or $\left.\mathbb{Z}_{N} \times \mathbb{Z}_{M}\right)$ and the pair $(i, j)$. The latter refer to the $i$ th torus lattice and the $j$ th rototranslation element, following the notation of Ref. [30]. The columns labeled by "No. models" display the number of SM-like models for the corresponding orbifold geometry.

\begin{tabular}{|c|c|c|c|c|c|c|c|c|c|}
\hline Orbifold & No. models & Orbifold & No. models & Orbifold & No. models & Orbifold & No. models & Orbifold & No. models \\
\hline $\mathbb{Z}_{2} \times \mathbb{Z}_{2}$ & & $\mathbb{Z}_{2} \times \mathbb{Z}_{4}$ & & $\mathbb{Z}_{2} \times \mathbb{Z}_{4}$ & & $\mathbb{Z}_{3} \times \mathbb{Z}_{3}$ & & $\mathbb{Z}_{\mathbf{3}}$ & \\
\hline$(1,1)$ & 3 & $(3,3)$ & 3935 & $(9,3)$ & 1491 & $(4,2)$ & 17 & $(1,1)$ & 155 \\
\hline$(2,1)$ & 8 & $(3,4)$ & 4779 & $(10,1)$ & 3562 & $(5,1)$ & 595 & $\mathbb{Z}_{4}$ & \\
\hline$(3,1)$ & 10 & $(3,5)$ & 9100 & $(10,2)$ & 3250 & $\mathbb{Z}_{3} \times \mathbb{Z}_{6}$ & & $(1,1)$ & 1 \\
\hline$(5,1)$ & 1 & $(3,6)$ & 1916 & $\mathbb{Z}_{2} \times \mathbb{Z}_{6}-\mathrm{I}$ & & $(1,1)$ & 43 & $(2,1)$ & 12 \\
\hline$(6,1)$ & 46 & $(4,1)$ & 1570 & $(1,1)$ & 109 & $(1,2)$ & 2 & $(3,1)$ & 17 \\
\hline$(7,1)$ & 145 & $(4,2)$ & 6179 & $(1,2)$ & 142 & $(2,1)$ & 98 & $\mathbb{Z}_{6}-\mathrm{I}$ & \\
\hline$(9,1)$ & 6 & $(4,3)$ & 3445 & $(2,1)$ & 45 & $(2,2)$ & 4 & $(1,1)$ & 33 \\
\hline$(10,1)$ & 5 & $(4,4)$ & 2905 & $(2,2)$ & 310 & $\mathbb{Z}_{4} \times \mathbb{Z}_{4}$ & & $(2,1)$ & 31 \\
\hline$(12,1)$ & 10 & $(4,5)$ & 3336 & $\mathbb{Z}_{2} \times \mathbb{Z}_{6}-\mathrm{II}$ & & $(1,1)$ & 234 & $\mathbb{Z}_{6}-$ II & \\
\hline $\mathbb{Z}_{2} \times \mathbb{Z}_{4}$ & & $(5,1)$ & 1771 & $(1,1)$ & 8 & $(1,2)$ & 90 & $(1,1)$ & 31 \\
\hline$(1,1)$ & 1779 & $(5,2)$ & 2413 & $(2,1)$ & 156 & $(1,3)$ & 637 & $(2,1)$ & 77 \\
\hline$(1,2)$ & 4590 & $(6,1)$ & 2648 & $(3,1)$ & 150 & $(1,4)$ & 5 & $(3,1)$ & 140 \\
\hline$(1,3)$ & 3117 & $(6,2)$ & 5542 & $(4,1)$ & 143 & $(2,1)$ & 300 & $(4,1)$ & 12 \\
\hline$(1,4)$ & 2215 & $(6,3)$ & 3726 & $\mathbb{Z}_{\mathbf{3}} \times \mathbb{Z}_{\mathbf{3}}$ & & $(2,2)$ & 172 & $\mathbb{Z}_{\mathbf{8}}-\mathrm{I}$ & \\
\hline$(1,5)$ & 9388 & $(6,4)$ & 3574 & $(1,1)$ & 10 & $(2,3)$ & 600 & $(1,1)$ & 4 \\
\hline$(1,6)$ & 7119 & $(6,5)$ & 1895 & $(1,2)$ & 71 & $(2,4)$ & 2 & $(2,1)$ & 4 \\
\hline$(2,1)$ & 1587 & $(7,1)$ & 1908 & $(1,3)$ & 3409 & $(3,1)$ & 339 & $\mathbb{Z}_{8}$-II & \\
\hline$(2,2)$ & 2886 & $(7,2)$ & 2322 & $(1,4)$ & 1843 & $(3,2)$ & 208 & $(1,1)$ & 330 \\
\hline$(2,3)$ & 6174 & $(7,3)$ & 612 & $(2,1)$ & 17 & $(4,1)$ & 1665 & $(2,1)$ & 93 \\
\hline$(2,4)$ & 9283 & $(8,1)$ & 4926 & $(2,2)$ & 521 & $(4,3)$ & 1 & $\mathbb{Z}_{12}$-IIō & \\
\hline$(2,5)$ & 2066 & $(8,2)$ & 3970 & $(2,3)$ & 6402 & $(5,1)$ & 579 & $(1,1)$ & 102 \\
\hline$(2,6)$ & 4029 & $(8,3)$ & 1919 & $(3,1)$ & 9 & $(5,2)$ & 2 & & \\
\hline$(3,1)$ & 2302 & $(9,1)$ & 2346 & $(3,2)$ & 1584 & $\mathbb{Z}_{6} \times \mathbb{Z}_{6}$ & & & \\
\hline$(3,2)$ & 5957 & $(9,2)$ & 464 & $(4,1)$ & 413 & $(1,1)$ & 12 & & \\
\hline
\end{tabular}

138 orbifold geometries of interest, as summarized in Table I. This enormous landscape of SM-like models invites us to pose questions that may hint towards fruitful corners in the landscape where the best phenomenology could emerge. Such questions include:

(1) What kind of exotic matter fields can we obtain from SM-like string models?

(2) Specifically, which of the leptoquarks introduced in the literature [43-45] (see also Sec. 95 of Ref. [46]) can be realized in this string setting?

(3) Are they useful for tackling some of the open questions of particle physics, such as the question of dark matter or the $g_{\mu}-2$ puzzle? (See also Refs. $[47,48]$ for other string approaches to explain the $g_{\mu}-2$ discrepancy within D-brane string compactifications.)

(4) Is the origin of the SM encoded in the properties of particular orbifold geometries?

In particular, the questions regarding leptoquarks are motivated by the recent enhancement of the $g_{\mu}-2$ anomaly, which has triggered a renewed interest in this area [49-57]. Additionally, leptoquarks have been long regarded as viable candidates for dark matter or solutions to some other flavor issues [58,59], though they are not completely free of challenges (see, e.g., Ref. [60]). We do no attempt to address the phenomenology of stringy leptoquarks. Our work establishes the foundation for future endeavors in this direction, which consists in describing what leptoquarks can be realized in string constructions. In this work, we provide some tools to address these questions by either systematically inspecting the properties of the identified models or applying machine learning techniques, as has been done recently in the SUSY case $[15,16,61-75]$.

The content of this work is structured as follows. In Sec. II we discuss the setting of our search for SM-like string models and provide an overview of our results. In Sec. III we analyze the massless spectra of our SM-like models in order to (i) identify the most promising cases, dubbed here almost $S M$, and (ii) uncover patterns in string theory that may lead to the best phenomenology. General features of the spectra of our models are deferred to the Appendixes. In Sec. IV we illustrate the qualities of our models by discussing some properties of a couple of sample models. Finally, in Sec. V we give our conclusions and outlook. 


\section{THE LANDSCAPE OF NONSUPERSYMMETRIC HETEROTIC ORBIFOLDS}

We consider the heterotic string without SUSY in $D=10$ with the gauge group $\mathrm{SO}(16) \times \mathrm{SO}(16)$. This theory can be obtained from the supersymmetric heterotic string with the gauge group $\mathrm{E}_{8} \times \mathrm{E}_{8}$ in the bosonic or fermionic formulation $[28,29]$. The ten-dimensional massless spectrum of this non-SUSY string theory is tachyon and anomaly free, and consists of 240 gauge bosons, 256 spinors, and 256 cospinors. The dilaton, graviton, and Kalb-Ramond field constitute its gravity sector.

In this work, in order to contrast SUSY and non-SUSY compactifications, we focus on the 138 orbifold geometries classified in Ref. [30]. That is, we follow the traditional prescription to arrive at 4D models by orbifold compactifications; see, e.g., Refs. [31,76,77]. In some detail, we define an orbifold geometry as the quotient

$$
\mathbb{O}=\frac{\mathbb{R}^{6}}{S},
$$

where $S$ is a so-called space group, whose elements are specified as $g=(\vartheta, \lambda)$. The so-called twists $\vartheta$ generate a (rotational) point group $P \subset \mathrm{O}(6)$, whereas $\lambda$ correspond to translations. Hence, space group elements act on the spatial coordinates $y \in \mathbb{R}^{6}$ of the extra dimensions according to

$$
y \stackrel{g}{\mapsto} \vartheta y+\lambda, \quad g \in S .
$$

In some cases, $\lambda$ is an element of the $6 \mathrm{D}$ torus lattice $\Lambda=\left\{n_{\alpha} e_{\alpha} \mid n_{\alpha} \in \mathbb{Z}, \alpha=1, \ldots, 6\right\}$, where $\left\{e_{\alpha}\right\}$ is the basis of $\Lambda$. Space group elements with $\lambda \notin \Lambda$ are called rototranslations. In the absence of rototranslations, the orbifold can also be defined as $\mathbb{O}=\mathbb{T}^{6} / P$, where $\mathbb{T}^{6}=\mathbb{R}^{6} / \Lambda$. It is evident that $P$ must then be a symmetry of $\Lambda$. Thus, in general, for each point group there are various orbifold geometries, as different $\Lambda$ can have the same point group symmetry. We are interested in toroidal orbifolds with and without rototranslations, where $P$ is Abelian. The details of the 138 space groups associated with $\mathbb{Z}_{N}$ and $\mathbb{Z}_{N} \times \mathbb{Z}_{M}$ point groups that we consider here were systematically obtained in Ref. [30] in the context of SUSY compactifications. These are equally useful to arrive at consistent $4 \mathrm{D}$ models from the non-SUSY heterotic string $\mathrm{SO}(16) \times$ $\mathrm{SO}(16)$. We shall explore all of them to find phenomenologically promising 4D non-SUSY models, which we call SM-like models.

Each orbifold geometry, characterized by a space group $S$, leads to a myriad of effective field theories in $4 \mathrm{D}$, with a given gauge group $\mathcal{G}_{4 \mathrm{D}}$ and massless spectrum of matter fields building representations of $\mathcal{G}_{4 \mathrm{D}}$, where we take all fermions to be left-chiral. These result from embedding the chosen orbifold geometry into the gauge degrees of freedom. These embeddings can be defined by a 16-dimensional (16D) shift vector $V_{i}$ for each rotational generator of the point group, and up to six 16D Wilson lines
$W_{\alpha}, \alpha=1, \ldots, 6$, subject to consistency conditions including especially modular invariance (see Sec. 3.2 of Ref. [31] and Ref. [78]). Standard techniques then yield the gauge groups and massless matter spectra on which we focus in this work.

We define a SM-like model by the following properties of the 4D gauge group and massless matter spectrum:

(1) $\mathcal{G}_{4 \mathrm{D}}=\mathcal{G}_{\mathrm{SM}} \times\left[\mathrm{U}(1)^{\prime}\right]^{n} \times \mathcal{G}_{\text {hidden }}$, where $\mathcal{G}_{\mathrm{SM}}=$ $\mathrm{SU}(3)_{C} \times \mathrm{SU}(2)_{L} \times \mathrm{U}(1)_{Y}$ is the $\mathrm{SM}$ gauge group, $\mathcal{G}_{\text {hidden }}$ is a non-Abelian gauge group, usually built as a product of $\mathrm{SU}(N)$ group factors, and $n>0$ is an integer number subject to the condition that $\operatorname{rank}\left(\mathcal{G}_{4 \mathrm{D}}\right)=16$. $\mathcal{G}_{\text {hidden }}$ is considered "hidden" because (almost) none of the SM fields are charged under this group.

(2) The 4D massless spectrum consists of exactly three generations of chiral fermions for quarks and leptons (including three right-handed neutrinos) and at least one Higgs doublet, a number of exotic fermions that are vector-like with respect to the SM, exotic scalars, and several SM-singlet scalars and fermions. In this way, all exotics can in principle be decoupled without breaking the SM gauge group.

The SM hypercharge is nonanomalous and compatible with $\mathrm{SU}(5)$ grand unification. In most cases, one of the additional $\mathrm{U}(1)^{\prime}$ appears anomalous (where the anomaly is canceled by the Green-Schwarz mechanism [79]). Note that an arbitrary number of (vector-like) exotics, Higgs doublets, and singlets arising from the compactifications are allowed for a SM-like model.

With the goal of performing an extensive search for SM-like models, we use the orbifolder, which we adapted to perform these non-SUSY compactifications. The orbifolder creates randomly and consistently the essential parameters to construct inequivalent and (perturbatively) tachyon-free SM-like models and computes their massless matter spectra. ${ }^{1}$ Using this tool and exploring all 138 orbifold geometries, we find SM-like models in 104 out of 138 orbifold geometries. Our results are presented in Table I. We find a total of 170219 inequivalent promising models, where 169177 (1042) belong to the $\mathbb{Z}_{N} \times \mathbb{Z}_{M}$ $\left(\mathbb{Z}_{N}\right)$ orbifold geometries. The largest number of SM-like models was found in the $\mathbb{Z}_{2} \times \mathbb{Z}_{4}$ ( $\mathbb{Z}_{8}$-II) orbifold geometries with 147996 (423) SM-like models, which reveals that both SUSY and non-SUSY promising orbifold compactifications are most abundant in these geometries; see, e.g., Refs. [14-16]. Our results represent, as far as we know, the most extensive search for SM-like models from string theory. Yet, our search is not exhaustive. In particular, about 1000 SM-like models with point groups $\mathbb{Z}_{8}$-I and

\footnotetext{
${ }^{1}$ Specifically, it creates string models by choosing shift vectors and Wilson lines that describe the geometrical orbifold action on the string's gauge degrees of freedom, and verifies their consistency under the worldsheet modular invariance conditions $[31,78]$.
} 
$\mathbb{Z}_{2} \times \mathbb{Z}_{2}$ were identified before in Ref. [31] and do not appear in our current search.

\section{EXPLORING THE SM-LIKE MODELS}

\section{A. Vectorlike exotics and Higgs doublets}

We are now interested in knowing explicitly the types of vector-like exotic (VLE) representations and the number of Higgses that appear in all 170219 identified SM-like models. The motivation of this study of the particle content is twofold. First, we aim at identifying the most promising SM-like candidates, i.e., those whose features best fit known observations. Second, among the VLE matter found in these constructions, inspecting the qualities of the leptoquark sector may be relevant for diverse phenomenological questions (see, e.g., Refs. $[49,52,58,59,80]$ ), including the recent enhancement of the muon $g_{\mu}-2$ anomaly.

Exotic matter refers to representations of $\mathcal{G}_{\mathrm{SM}}=$ $\mathrm{SU}(3)_{C} \times \mathrm{SU}(2)_{L} \times \mathrm{U}(1)_{Y}$ appearing in the 4D massless spectrum of an orbifold compactification, beyond the three generations of SM fermions, including three right-handed neutrinos, and one Higgs doublet. Further, to be characterized as vector-like, (i) each exotic fermion must be accompanied by another fermion with the exact opposite charges, or (ii) it must be a scalar. In a slight abuse of the notation, we shall also count additional fermionic singlets under $\mathcal{G}_{\mathrm{SM}}$ and scalar SM singlets as VLE matter. The former may play the role of sterile right-handed neutrinos (see, e.g., Ref. [20] for its SUSY equivalent), and the latter can be regarded as scalar dark matter candidates in the framework of Higgs portals $[81,82]$ or also flavon fields in the Froggatt-Nielsen mechanism $[83,84]$. We shall refer to the latter simply as flavons here.

We report our findings on the different types of exotics in Tables II-V of Appendix A. We list all types of VLE matter representations with respect to the SM gauge group. We find 26 kinds of VLE fermions and another 26 representations for exotic scalars. Tables II and III show the percentage of models that exhibit any of the different exotic fermion or scalar representations. We observe that fermion and scalar singlets are always present in all of the models from $\mathbb{Z}_{N}$ and $\mathbb{Z}_{N} \times \mathbb{Z}_{M}$ orbifolds. Tables IV and V present the average numbers of exotic fermions and scalars, respectively.

As a sample case, consider the 155 SM-like models that arise from orbifolds with the $\mathbb{Z}_{3}$ point group. The second column of Table II shows that the only exotic fermions with standard SM quantum numbers are down-type quark singlets and lepton doublets, which appear in most of these models. There are on average about three of these states, as we can see in Table IV. Further, about half of the models exhibit many kinds of fractionally charged fermions [85]. Concerning the exotic scalars, in Table III we observe that $\mathbb{Z}_{3}$ SM-like models generically exhibit various types of (scalar) leptoquarks. In the notation of Ref. [44], we identify the leptoquarks $\tilde{R}_{2}:(\mathbf{3}, \mathbf{2})_{1 / 6}$ and $\bar{S}_{1}:(\overline{\mathbf{3}}, \mathbf{1})_{-2 / 3}$ in about half of the $\mathbb{Z}_{3}$ SM-like models, and $S_{1}:(\overline{\mathbf{3}}, \mathbf{1})_{1 / 3}$ in all models of this orbifold geometry. We see in Table $V$ that they are not very abundant in these models: there are on average $\sim 1.5$ leptoquarks $\tilde{R}_{2}$ and $\bar{S}_{1}$, while the mean value of the multiplicity of $S_{1}$ leptoquarks is about 5.6 in these models.

Interestingly, our tables reveal that the scalar leptoquarks $S_{1}, \bar{S}_{1}$, and $\tilde{R}_{2}$ identified in the $\mathbb{Z}_{3}$ example are generic in all SM-like orbifold models. No other leptoquarks appear. As we shall shortly see, the existence of these leptoquarks might be related to a string-specific structure of localized strings in extra dimensions related to an $\mathrm{SU}(5)$ grand unification - so-called local grand unified theories (GUTs).

From Tables III and V, we note that there is a large number of scalar fields with SM quantum numbers $(\mathbf{1}, \mathbf{2})_{1 / 2}$. These fields correspond to Higgs doublets in our SM-like models. Thus, we find different extensions of the SM with various numbers (from 1 to 55) of Higgs doublets, such as those previously studied from a bottom-up perspective [86-88]. For the different point groups of our orbifold geometries, we display in Table VI the number of Higgses we find in all 170 219 SM-like models. We see that only the $\mathbb{Z}_{6}$-I orbifold geometries yield models with just one Higgs doublet (in 13 out of 64 models). There are 3192 SM-like models with two Higgs doublets distributed in the $\mathbb{Z}_{8}$-II, $\mathbb{Z}_{2} \times \mathbb{Z}_{4}, \mathbb{Z}_{2} \times \mathbb{Z}_{6}$-I, and $\mathbb{Z}_{3} \times \mathbb{Z}_{3}$ orbifold geometries. Higher multiplicities of Higgs doublets seem to be favored in our constructions, where most models are endowed with 11,9 , or 15 Higgs doublets (20378, 16657, and 16485 SM-like models in each case). Although no extra Higgs fields have been observed, they might have interesting implications, especially for dark matter and Higgs phenomenology [89-91], and for an explanation of the $g_{\mu}-2$ tension [92-96].

As we show in Appendix $\mathrm{C}$, there are high correlations between the numbers of different VLE representations appearing in our matter spectra. In particular, we find almost perfect correlations among the scalar leptoquarks $\bar{S}_{1}, \tilde{R}_{2}$ and the charged scalars $(\mathbf{1}, \mathbf{1})_{1}$. Further, the appearance of leptoquarks $S_{1}$ and extra Higgs doublets $\phi:(\mathbf{1}, \mathbf{2})_{1 / 2}$ is correlated, too. We note that a scalar 10-plet of SU(5) precisely decomposes as $\mathbf{1 0}=\tilde{R}_{2} \oplus \bar{S}_{1} \oplus(\mathbf{1}, \mathbf{1})_{1}$, while a scalar 5-plet is built by $\mathbf{5}=S_{1}^{*} \oplus \phi$, where $S_{1}^{*}$ is the complex-conjugated scalar. ${ }^{2}$ Thus, the observed correlations suggest a common origin for these fields. It is known that heterotic orbifolds can produce so-called local GUTs [12,97-99]. In these scenarios, the gauge symmetry is enhanced to an SU(5) GUT not in $4 \mathrm{D}$, but locally in extra dimensions at the orbifold singularities, where full GUT multiplets are realized. Hence, the number of leptoquarks seems to be related to these local GUTs.

\footnotetext{
${ }^{2}$ Here, we use the fact that scalars have no chirality. Thus, it is just a matter of convention to denote the scalar field that originates from the string in the representation $(\overline{\mathbf{3}}, \mathbf{1})_{1 / 3}$ as either $S_{1}$ or $S_{1}^{*}$, such that either $S_{1}^{*}$ or $S_{1}$ transforms as the complexconjugated representation $(\mathbf{3}, \mathbf{1})_{-1 / 3}$.
} 
Finally, we compare our findings on VLE with previous results in the context of minimal supersymmetric SM (MSSM)-like models that result from heterotic orbifold compactifications [16]. We see a few differences. The most evident difference is that, in general, SUSY models seem to produce more exotic representations than our SM-like models. However, exotic fermions with charges $(\overline{\mathbf{3}}, \mathbf{2})_{5 / 6}$ show up in our models (cf. Table II of Appendix A), but do not appear in MSSM-like orbifold models. In contrast, we observe several similarities between the massless spectra of MSSM-like and SM-like string models: there are roughly between 50 and 200 SM singlets (neutrinos and flavons); the most common exotics with SM quantum numbers are $(\overline{\mathbf{3}}, \mathbf{1})_{1 / 3}$ and $(\mathbf{1}, \mathbf{2})_{-1 / 2}$, suggesting a local GUT picture with $\overline{5}$-plets of SU(5) localized at some orbifold singularities, as mentioned before. Following the classification of Ref. [44] and considering SUSY breakdown in MSSM-like models, the only possible leptoquarks in all semirealistic models are just $S_{1}, \bar{S}_{1}$, and $\tilde{R}_{2}$, and the most common fractionally charged fields have SM charges $(\mathbf{1}, \mathbf{1})_{1 / 2}$ and $(\mathbf{1}, \mathbf{2})_{0}$.

\section{B. Almost SM models from heterotic orbifolds}

We would now like to identify in the landscape of nonSUSY heterotic orbifolds the models that best reproduce the particle content of the SM. With this purpose, we systematically inspect the spectra of our models to select those that contain three SM generations of fermions and the standard Higgs doublet, along with the least amount of exotic matter. Since SM singlets could play an important phenomenological role either as extra sterile neutrinos if they are fermions, or flavons or dark matter if they are scalars, we shall not count them as exotic states here.

The special SM-like models whose massless matter spectra display the closest resemblance with the SM are here called almost SM. These models can be classified into two categories:

(1) Models with no exotic fermions. We find 45 almost $S M$ models of this kind, distributed in the orbifold geometries $\mathbb{Z}_{2} \times \mathbb{Z}_{4}(1,6), \mathbb{Z}_{2} \times \mathbb{Z}_{4}(2,4)$, and $\mathbb{Z}_{3} \times$ $\mathbb{Z}_{3}(1,4)$, as summarized in Table VII. The spectra of these models include, besides the three SM generations, only $S_{1}$ scalar leptoquarks accompanied by various numbers of extra Higgs doublets, righthanded neutrinos, and SM-singlet scalars. As we see in Table VIII, we find that there are only three of these models with the minimal number of Higgs doublets (six) in this category.

(2) Models with no exotic scalars. There are 502 almost $S M$ models of this category, distributed in ten different $\mathbb{Z}_{2} \times \mathbb{Z}_{4}$ orbifold geometries. As displayed in Table VII, the scalar sector of their spectra include six Higgs doublets (one of them would be the standard Higgs) and SM-singlet scalars. In the fermionic sector, beyond several right-handed neutrinos, the only exotic fermions have quantum numbers $(\overline{\mathbf{3}}, \mathbf{1})_{1 / 3}$ and $(\mathbf{1}, \mathbf{2})_{-1 / 2}$ (plus their complex conjugates), mostly originated from full multiplets of SU(5) local GUTs. Additional details, such as the shift vectors and Wilson lines of these selected 547 almost SM models, can be found on our website [100] in a format compatible with the (nonSUSY) orbifolder.

Some comments are in order. First, there is no SM-like model without exotics. Second, the existence of a few exotics in the models identified as almost $S M$ might be phenomenologically challenging. For example, the $S_{1}$ scalar leptoquarks of models without exotic fermions could lead to rapid proton decay if their couplings with firstgeneration quarks are unsuppressed and they do not develop very large masses. Fortunately, in principle, all leptoquarks in this case and all exotic fermions in the second category of almost SM models can be decoupled, when some flavons attain vacuum expectation values (VEVs). The details of this mechanism are beyond the scope of this paper and shall be discussed elsewhere.

As a last comment, let us mention the possibility of SM-like models with only one Higgs doublet; see Ref. [31]. We only find seven [six] models of this type, arising from the $\mathbb{Z}_{6}-\mathrm{I}(1,1)\left[\mathbb{Z}_{6}-\mathrm{I}(2,1)\right]$ orbifold geometries. Unfortunately, as shown in Table VII, these models include several VLE. In the scalar sector, besides 71 flavon or dark matter candidates on average, they include two $S_{1}$ leptoquarks and the exotic representations $(\mathbf{3}, \mathbf{1})_{1 / 6},(\mathbf{1}, \mathbf{2})_{0}$ and $(\mathbf{1}, \mathbf{1})_{1 / 2}$. In the fermionic sector, in addition to about 200 right-handed neutrinos, we see extra vector-like pairs of down-type quark singlets and lepton doublets, as well as fractionally charged exotics in the representations $(\mathbf{3}, \mathbf{1})_{1 / 6}$, $(\mathbf{1}, \mathbf{2})_{0}$ and $(\mathbf{1}, \mathbf{1})_{1 / 2}$ (paired up with their complex conjugates). Although the exotics could in principle be decoupled from the low-energy effective theory, these models seem to be in worse shape than our almost SM.

\section{Predicting the stringy origin of the SM with machine learning}

The previous observations based on a systematic (though limited) search reveal some of the general properties of the matter spectra of a subset of all possible SM-like string models. Fortunately, by using machine learning (ML) techniques, as in Ref. [16], we can learn more.

Here, based on the information provided by the identified models, we obtain an ML algorithm that predicts the specific orbifold geometry that most likely hosts a SM-like string model with a given particle content of exotics. We address this task using supervised machine learning and evaluate the quality of our algorithms using the accuracy and the f1-macro. The accuracy of our predictive ML algorithm is given by the number of correct predictions divided by the total number of predictions. On the other hand, the f1-macro is computed as the average of the f1-scores of each of the 104 orbifold geometries. Since our data set is imbalanced, the f1-macro is more suitable for our task; see, for example, Sec. 3.1 of Ref. [16]. 


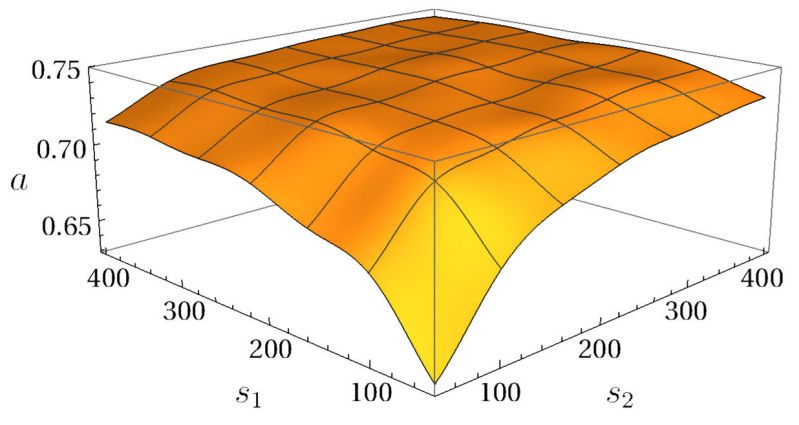

(a)

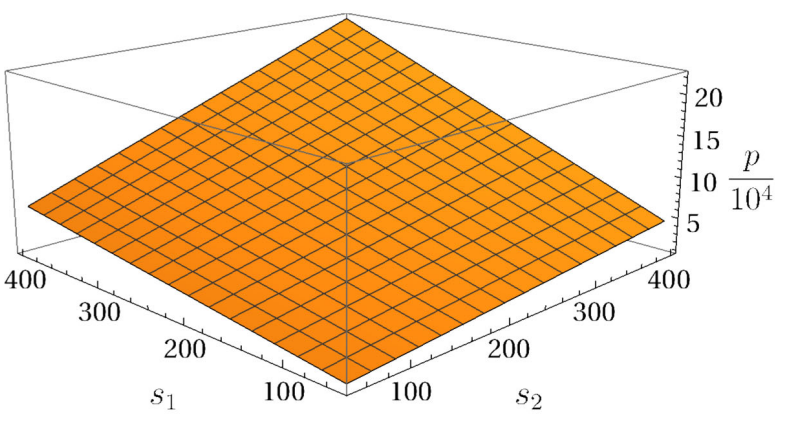

(b)

FIG. 1. (a) Accuracies $a$ of the validation set and (b) number of trainable parameters $p$ for neural networks with two hidden layers of sizes $s_{1} \in\{50,100, \ldots, 400\}$ and $s_{2} \in\{50,100, \ldots, 400\}$.

In order to compare to the accuracy and the f1-macro of a "good" ML algorithm, we first compute the so-called null value. The null value is based on the trivial algorithm that always predicts the orbifold geometry that appears most frequently in our data set of 170219 inequivalent SM-like models, independently of the given particle spectrum. In our data set, $\mathbb{Z}_{2} \times \mathbb{Z}_{4}(1,5)$ is the orbifold geometry that yields the largest number of SM-like models, with a total number of 9388 SM-like models. Hence, we can estimate the accuracy of the trivial algorithm based on our data set: it gives a correct prediction with a probability of $5.5 \%$, i.e.,

$$
\text { null value accuracy: } \frac{9,388}{170219} \approx 5.5 \% \text {. }
$$

In addition, we compute the f1-scores and the f1-macro for the trivial algorithm. We obtain $\mathrm{f} 1(\mathbb{O})=0$ for $\mathbb{O} \neq \mathbb{Z}_{2} \times$ $\mathbb{Z}_{4}(1,5)$ and $\mathrm{f} 1(\mathbb{O})=1$ for $\mathbb{O}=\mathbb{Z}_{2} \times \mathbb{Z}_{4}(1,5)$. Hence,

$$
\text { null value f1-macro: } \frac{1+0+\cdots+0}{104} \approx 1 \% \text {. }
$$

These are our null values against which we will compare our results in the following.

Before we discuss our ML algorithm, we first split our data set into $80 \%$ training data and $20 \%$ test data. Since the label of the orbifold geometry is a categorical data (i.e., data without ordering), we use a one-hot encoding for the labels of the 104 orbifold geometries that host SM-like string models. Furthermore, for each SM-like string model, we represent the exotic particle spectrum by a 52-dimensional vector of integers (for the 26 fermionic and 26 bosonic exotics as listed in Tables II and III). Hence, our ML algorithm $f_{\mathrm{ML}}$ takes a 52-dimensional vector $X \in \mathbb{N}^{52}$ as input (corresponding to the particle spectrum of exotics) and gives a 104-dimensional vector as output (corresponding to the one-hot encoded prediction of the orbifold geometry that most likely can produce the given particle spectrum),

particle spectrum of exotics $X \stackrel{f_{\text {ML }}}{\mapsto}$ orbifold geometry $\mathbb{O}$.
As our ML algorithm, we take a fully connected neural network. The input layer has 52 nodes corresponding to $X$, and the output layer has 104 nodes (corresponding to the 104 orbifold geometries). We add two hidden layers with $s_{1}$ and $s_{2}$ nodes, respectively. Then, the number of trainable parameters of the neural network is given by

$$
p=53 s_{1}+105 s_{2}+s_{1} s_{2}+104
$$

[see Fig. 1(b)], and we want to balance between the accuracy of our neural network and the number of trainable parameters. As activation functions we choose "selu" except for the output layer. There, we use the "softmax" activation functions, such that each value in the output layer lies in the range $[0,1]$ and the sum of output values is normalized to 1 . Then, we can interpret the $i$ th output value as the probability that the $i$ th orbifold geometry can reproduce the given particle spectrum. In addition, we use a learning rate of 0.001 and the loss is computed using “categorical_crossentropy." Using our training set, we scan over network architectures with

$s_{1} \in\{50,100, \ldots, 400\}$ and $s_{2} \in\{50,100, \ldots, 400\}$,

using a $20 \%$ validation split and train each neural network three times for 200 training epochs. The averaged maximal accuracies of the validation set are evaluated and plotted in Fig. 1(a). The best accuracy of the validation set is around $75 \%$ for a network architecture with $s_{1}=300$ and $s_{2}=$ 400 (with 178004 trainable parameters). However, using $s_{1}=100$ and $s_{2}=350$ (with 77154 trainable parameters) we already obtain an accuracy of $74 \%$ (and the f1-macro of the validation set is $73 \%$ ). Thus, we choose the smaller but almost equally good network architecture. After $\approx 120$ epochs of training the loss of the validation set starts to increase; see Fig. 2(a). Hence, the neural network begins to overfit. Thus, we stop training after 120 training epochs. Then, we construct and train 21 neural networks of this architecture and use a majority vote of the 21 individual predictions to obtain a final prediction. By doing so, the accuracy of the test set (consisting of $20 \%$ of all data) increases slightly to $76 \%$. We display the confusion matrix of the test set as a heat map in Fig. 2(b). 


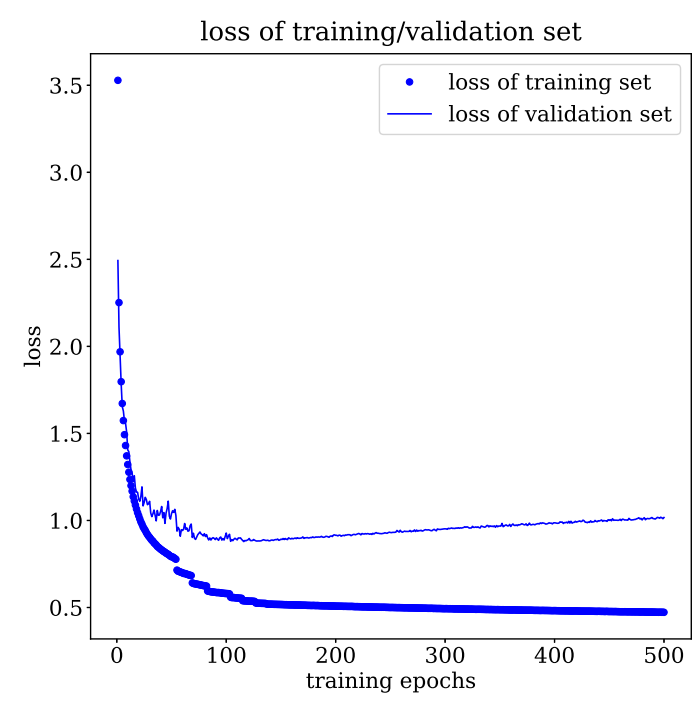

(a)

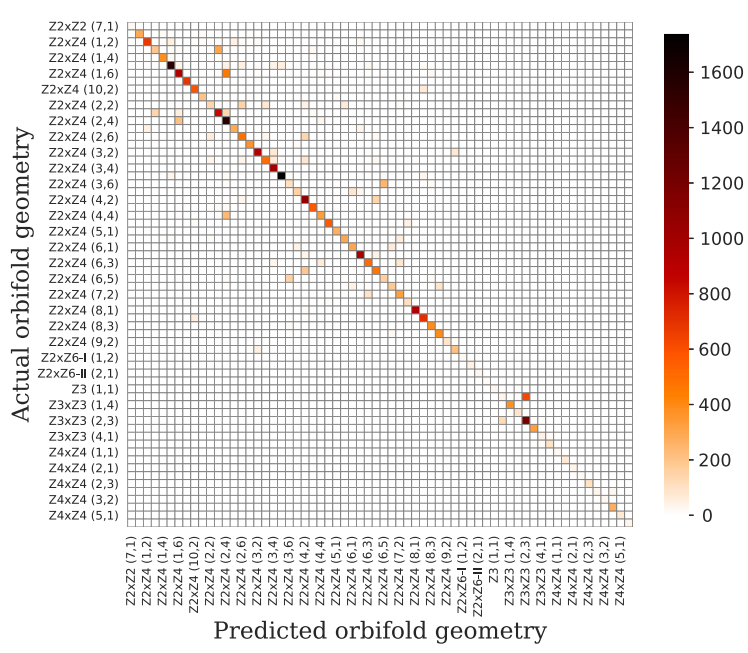

(b)

FIG. 2. (a) Loss of training and validation sets after a certain number of training epochs of our best neural network architecture with $s_{1}=100$ and $s_{2}=350$. Note that the loss of the validation set increases after $\approx 120$ epochs, which signals overfitting. (b) Using a majority vote of 21 neural networks of the size $s_{1}=100$ and $s_{2}=350$, we compute the confusion matrix of the test set and display it as a heat map (restricted to those orbifold geometries that have at least 50 SM-like models in the test set).

Now, we can use our trained neural networks to extrapolate to SM-like models that have not been discovered in the string landscape so far. By giving a spectrum of exotics to the trained neural networks, we obtain a prediction for the orbifold geometry that most likely can host this model. For example, we ask the networks what the orbifold geometry is that can most likely reproduce the exact SM spectrum without charged exotics. In detail, we specify a SM spectrum that contains in addition to the Higgs and the three generations of quarks and leptons only SM singlets: a (large) number of right-handed neutrinos (which can be utilized for an extended seesaw mechanism; see Ref. [20]) and a (large) number of SM scalar singlets. The results are visualized in Fig. 3. For certain numbers of singlets the orbifold geometries $\mathbb{Z}_{2} \times \mathbb{Z}_{2}(12,1), \mathbb{Z}_{3} \times \mathbb{Z}_{3}$ $(1,4)$, and $\mathbb{Z}_{2} \times \mathbb{Z}_{4}(1,6)$ are predicted to be able to reproduce these spectra.

By comparing to our 170219 explicitly constructed SMlike models, we see that in particular the orbifold geometries $\mathbb{Z}_{2} \times \mathbb{Z}_{2}(12,1)$ and $\mathbb{Z}_{3} \times \mathbb{Z}_{3}(1,4)$ are able to produce the SM spectrum with the least number of additional particles compared to all other orbifold geometries. In addition, we predict the orbifold origin of SM-like models with 110 SM scalar singlets (which roughly corresponds to the average number of singlets over all orbifold geometries; see Table V) and with some scalar leptoquarks $S_{1}, \bar{S}_{1}$, and $\tilde{R}_{2}$, but no further exotics. The results are illustrated in Figs. 4(a)-4(c). Finally, we predict the orbifold origin of a SM-like model with no other exotics except for a (large) number of additional Higgs doublets; see Fig. 4(d). Recall that we have found correlations between the number of leptoquarks $\bar{S}_{1}$ and $\tilde{R}_{2}$, and between the number of leptoquarks $S_{1}$ and the number of additional Higgs doublets; see the respective plots in Fig. 5. Therefore, it is also clear why the predictions in Figs. 4(a) and 4(b) are very similar, as well as the predictions in Figs. 4(c) and 4(d).

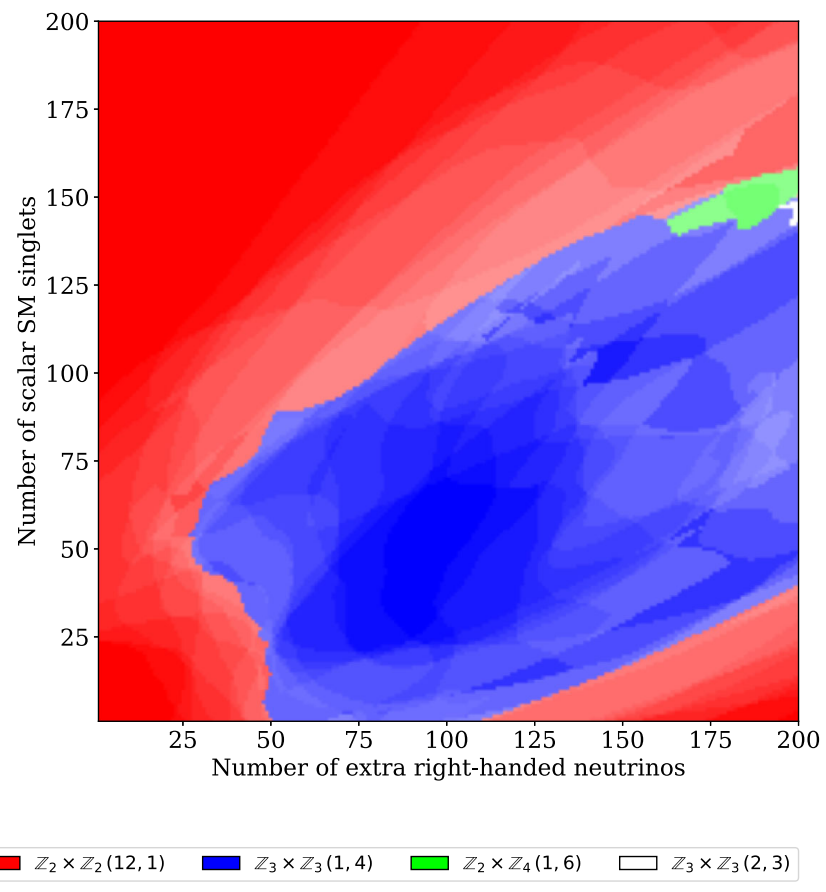

FIG. 3. Predictions of orbifold geometries for SM-like spectra without exotics, except for $[1, \ldots, 200]$ additional right-handed neutrinos and $[1, \ldots, 200]$ scalar flavons $(\mathbf{1}, \mathbf{1})_{0}$. The transparency of each pixel indicates the accuracy of the respective prediction: the less transparent the color, the more accurate the prediction. 
(a)
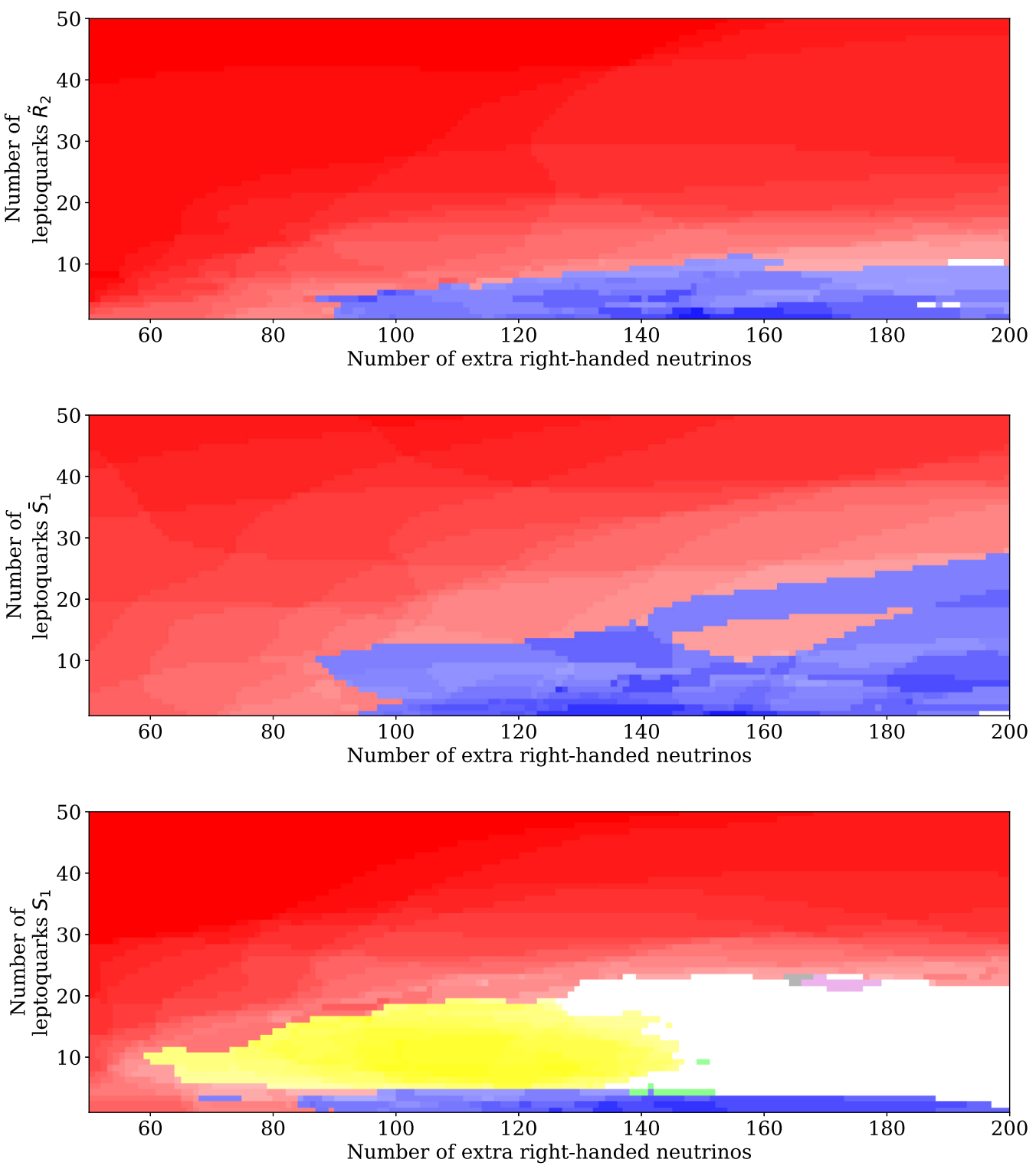

(d)

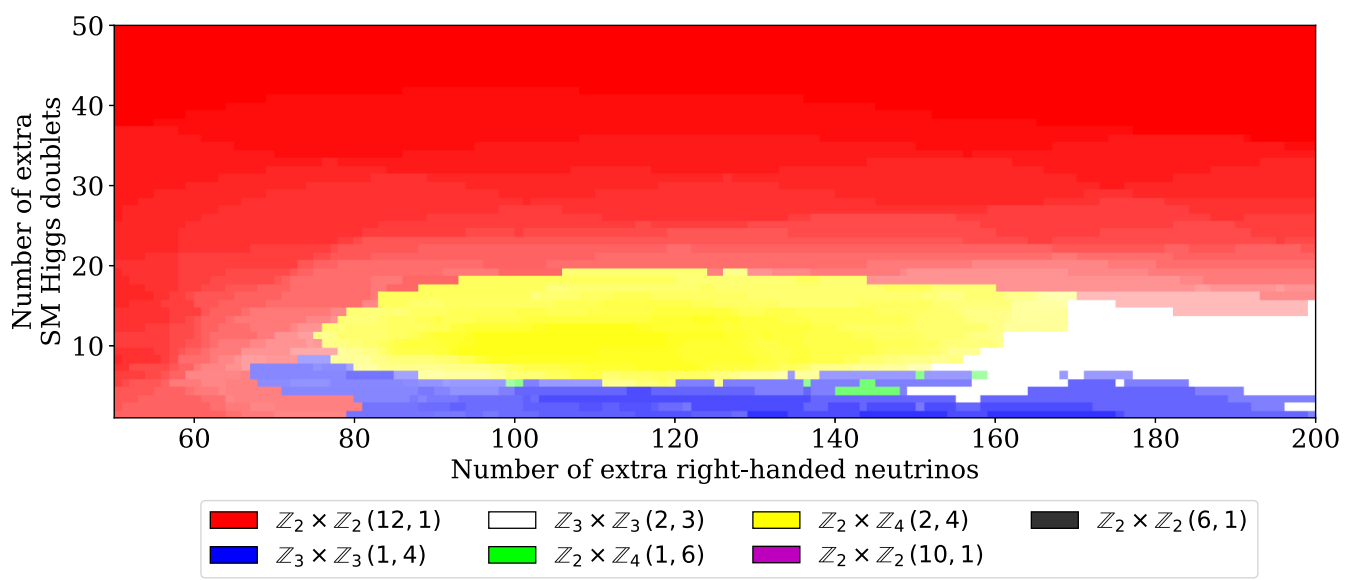

FIG. 4. Predictions of orbifold geometries for SM-like spectra without exotics, except for 110 scalar flavons $(\mathbf{1}, \mathbf{1})_{0}$, and additional particles as indicated by the labels of the axes. The transparency of each pixel indicates the accuracy of the respective prediction: the less transparent the color, the more accurate the prediction. 


\section{BENCHMARK SM-LIKE MODELS}

In this section we discuss some details of three benchmark SM-like string models. Two of them are characteristic almost SM orbifold models: one without exotic scalars and one without exotic fermions. The third model is a SM-like model including a small number of right-handed neutrinos and SM-singlet scalars. These models arise from different $\mathbb{Z}_{N} \times \mathbb{Z}_{M}$ orbifold geometries. Hence, they are defined by these geometries and their gauge embedding in terms of the $16 \mathrm{D}$ shift vectors $V_{1}, V_{2}$ and Wilson lines $W_{\alpha}, \alpha=1, \ldots, 6$.

Our benchmark models are defined as follows:

(1) Model 1. Almost SM model based on the orbifold geometry $\mathbb{Z}_{2} \times \mathbb{Z}_{4}(2,4)$ and its gauge embedding given by the shift vectors

$$
\begin{aligned}
V_{1}=\frac{1}{2} & (0,0,0,0,0,1,1,4,0,0,0,0,1,1,1,1), \\
V_{2}= & \frac{1}{8}(-3,-1,-1,-1,3,1,9,-3,-5, \\
& \quad-1,1,1,-1,-1,1,5),
\end{aligned}
$$

and the Wilson lines (with $W_{1}=W_{2}=0$ )

$$
\begin{aligned}
W_{3}= & W_{4}=W_{6}=\frac{1}{2}(0,0,0,0,0,0,0,0,-1, \\
& 0-3,0-3,2,-2,3), \\
W_{5}= & \frac{1}{4}(-7,-1,-1,3,7,-3,9,-7,-7, \\
& 5,-5,9,-3,7,7,3) .
\end{aligned}
$$

The resulting $4 \mathrm{D}$ gauge group is given by

$\mathcal{G}_{4 D}=\mathrm{SU}(3)_{C} \times \mathrm{SU}(2)_{L} \times \mathrm{U}(1)_{Y} \times \mathcal{G}_{\text {hidden }} \times \mathrm{U}(1)^{\prime 8}$,

where $\mathcal{G}_{\text {hidden }}=\mathrm{SU}(3) \times \mathrm{SU}(2)^{2}$ is the hidden gauge group and one of the $\mathrm{U}(1)^{\prime}$ is anomalous. The SM gauge quantum numbers of the massless spectrum are presented in Table IX, where we explicitly display the pairs of vector-like exotic fermions. Note that there are three vector-like pairs of extra lepton doublets and four pairs of exotic down-type quarks, which can develop large masses when some of the 76 flavons attain VEVs. This model exhibits a total of six Higgs doublets and 111 right-handed neutrinos.

(2) Model 2. Almost SM model based on the orbifold geometry $\mathbb{Z}_{3} \times \mathbb{Z}_{3}(1,4)$ and its gauge embedding given by the shift vectors

$$
\begin{aligned}
V_{1}= & \frac{1}{6}(-9,-1,-1,-1,-1,1,1,3 \\
& -1,1,1,1,1,1,1,7)
\end{aligned}
$$

$$
\begin{aligned}
V_{2}= & \frac{1}{6}(9,-11,-3,1,1,-5,3,-11,-1,-11, \\
& -1,-1,-1,1,3,3),
\end{aligned}
$$

and the Wilson lines (with $W_{\alpha}=W_{1}$ for all $\alpha$ )

$$
\begin{aligned}
W_{1}= & \frac{1}{6}(-1,5,-9,-1,3,-9,-9,-11,-11, \\
& -7,-1,1,5,-1,-11,13) .
\end{aligned}
$$

The 4D gauge group reads

$$
\mathcal{G}_{4 D}=\mathrm{SU}(3)_{C} \times \mathrm{SU}(2)_{L} \times \mathrm{U}(1)_{Y} \times \mathcal{G}_{\text {hidden }} \times \mathrm{U}(1)^{\prime 9},
$$

where $\mathcal{G}_{\text {hidden }}=\mathrm{SU}(2)^{3}$ is the hidden gauge group and one of the $\mathrm{U}(1)^{\prime}$ is anomalous. The SM gauge quantum numbers of the massless spectrum are shown in Table IX. As exotics, this model includes nine $S_{1}$ leptoquarks and eight additional Higgs doublets. Clearly, including the standard Higgs doublet, these fields build 5-plets of local SU(5) GUTs in higher dimensions. In addition, we observe a large set of right-handed neutrinos and 30 scalar singlets.

(3) Model 3. SM-like string model based on the orbifold geometry $\mathbb{Z}_{2} \times \mathbb{Z}_{2}(12,1)$ and its gauge embedding given by the shift vectors

$$
\begin{aligned}
V_{1}= & \frac{1}{2}(0,0,0,0,0,0,0,8,-2,0,0,0,0,0,1,1), \\
V_{2}= & \frac{1}{4}(-1,-1,1,1,1,1,19,7,-5,-1,-1, \\
& -1,-1,5,-7,-1),
\end{aligned}
$$

and the Wilson lines (with $W_{1}=W_{2}=W_{3}$ and $\left.W_{4}=W_{5}=W_{6}\right)$

$$
\begin{aligned}
W_{1}= & \frac{1}{8}(1,13,-1,-1,-1,15,9,21,7,-1, \\
& -1,1,3,-11,7,15), \\
W_{4}= & \frac{1}{4}(6,2,-1,0,1,-6,-3,1,0,0,0,0,0,0,0,0) .
\end{aligned}
$$

The $4 \mathrm{D}$ gauge group is given by

$$
\mathcal{G}_{4 D}=\mathrm{SU}(3)_{C} \times \mathrm{SU}(2)_{L} \times \mathrm{U}(1)_{Y} \times \mathcal{G}_{\text {hidden }} \times \mathrm{U}(1)^{\prime 10},
$$

where $\mathcal{G}_{\text {hidden }}=\mathrm{SU}(2)^{2}$ is the hidden gauge group and one of the $\mathrm{U}(1)^{\prime}$ is anomalous. The SM quantum numbers of the matter spectrum of this model are displayed in Table IX. We observe that this model yields the smallest number of SM singlets among the 
spectra of the benchmark models. However, there is a large number of (pairs of) vector-like exotic fermions and $S_{1}$ leptoquarks. As in many other SM-like models, there are six Higgs doublets.

\section{CONCLUSIONS AND OUTLOOK}

In this work we have performed the most extensive search for SM-like models from orbifold compactification of the non-SUSY heterotic string $\mathrm{SO}(16) \times \mathrm{SO}(16)$. We inspected their massless spectra looking for the SM-like models whose spectra best resemble the one of the SM, and for useful patterns that may guide us to find the SM from string theory.

Using a non-SUSY extension of the orbifolder and considering all 138 orbifolds classified in Ref. [30], we found 170219 SM-like models distributed among 104 orbifold geometries, as presented in Table I. Orbifolds with point groups $\mathbb{Z}_{2} \times \mathbb{Z}_{4}$ and $\mathbb{Z}_{8}$-II produce the majority of the models, with 147996 out of 169177 in $\mathbb{Z}_{N} \times \mathbb{Z}_{M}$ orbifolds and 423 out of 1042 in $\mathbb{Z}_{N}$ orbifolds. These models include the SM gauge group, three generations of SM fermions, including three right-handed neutrinos, at least one Higgs doublet, a number of SM-singlet scalars or fermions, and a few vector-like exotic fermions and exotic scalars. We classified all (52) possible exotic representations (where some of them behave as leptoquarks), and the number (1-55) of Higgs doublets that can appear in SMlike string models. Our results, summarized in the tables of Appendix A, indicate that only certain types of exotic representations appear in these constructions and they are not arbitrary. In particular, they generically build representations of $\mathrm{SU}(5)$ (local) GUTs at the singularities of the orbifold in extra dimensions. Further, most exotic scalars transform either as extra Higgs doublets or as $S_{1}, \bar{S}_{1}$, or $\tilde{R}_{2}$ scalar leptoquarks; see Ref. [44] for notation and Refs. $[49,52,58,59,80,101]$ for their phenomenology.

We explored the massless spectra of our SM-like models in order to identify special SM-like string models called here almost SM models and exhibiting either (i) no exotic fermions or (ii) no exotic scalars, except for SM singlets that may play the role of right-handed neutrinos in the fermionic sector and flavons or dark matter candidates in the scalar sector. The details of these models were discussed in Sec. III B and summarized in the tables Appendix B.

In Sec. III C we applied machine learning techniques to our data set of 170219 SM-like string models. Following Ref. [16], we trained a neural network such that it predicts, based on a requested particle spectrum, the orbifold geometry that most likely can host the corresponding SM-like string model. Our analysis shows that the underlying orbifold geometry leaves a distinct imprint on the matter spectrum of the resulting SM-like string model. We were thus able to predict the phenomenologically most promising orbifold geometries to be $\mathbb{Z}_{2} \times \mathbb{Z}_{2}$ $(12,1), \mathbb{Z}_{3} \times \mathbb{Z}_{3}(1,4), \mathbb{Z}_{2} \times \mathbb{Z}_{4}(2,4)$, and $\mathbb{Z}_{3} \times \mathbb{Z}_{3}(2,3)$; see Figs. 3 and 4. Note that we have made the list of all particle spectra available and invite the community to consider them in their studies. This information can be found on our website [100]. Our data includes (i) the files that contain shifts and Wilson lines of all 170219 SM-like string models, (ii) files that contain the almost SM models, and (iii) the massless matter spectra for all 170219 SM-like models.

To illustrate the qualities of our models, in Sec. IV we presented three special models: two almost $S M$ models and one SM-like model with a reduced number of SM singlets. They correspond to a sample of the models that arise from the three most promising orbifold geometries identified by using machine learning techniques.

One task beyond this work is the detailed study of the phenomenology of our SM-like string models. With this purpose, one should first construct the interaction terms $\mathcal{L}_{\text {int }}$ that give rise to the couplings among the different SM fields and the (scalar and fermionic) exotics. From this, one could obtain constraints on the parameters of the couplings that may lead to rapid proton decay, that could explain the $g_{\mu}-2$ discrepancy via leptoquarks, or that could provide admissible scenarios of multi-Higgs portals to dark matter, among other scenarios. Some of these phenomenological questions shall be studied elsewhere.

Another interesting endeavor in the context of nonSUSY heterotic orbifold compactifications is the study of the emerging eclectic flavor scheme, which is the natural nontrivial combination of traditional and modular flavor symmetries appearing in string orbifolds [102-104]. The eclectic picture has been studied only in the supersymmetric context, i.e., in the case of orbifold compactifications of the $E_{8} \times E_{8}$ heterotic string. As modular and traditional flavor symmetries originate from the outer automorphisms of the Narain space group, which are independent of the presence of supersymmetry, extending the discussion to the non-SUSY case should be feasible. This would, on the one hand, provide an understanding of modular flavor symmetries without SUSY, and on the other hand, complete the classification of all possible flavor symmetries emerging from orbifold compactifications $[14,105]$.

The final goal of the construction of nonsupersymmetric string models is to arrive at a phenomenologically viable model. This requires addressing the questions of potential instabilities beyond the perturbatively tachyon-free spectra presented here and the potentially large cosmological constant, as discussed in, e.g., Refs. [37,38,106-111]. We postpone the study of these challenges for future works.

\section{ACKNOWLEDGMENTS}

P. V. is supported by the Deutsche Forschungsgemeinschaft (SFB1258). We would like to thank J. Armando Arroyo, Esaú Cervantes, Yessenia Olguín-Trejo, and Omar Pérez-Figueroa for useful discussions. 


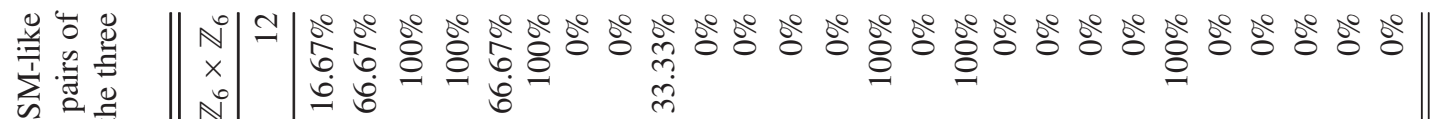

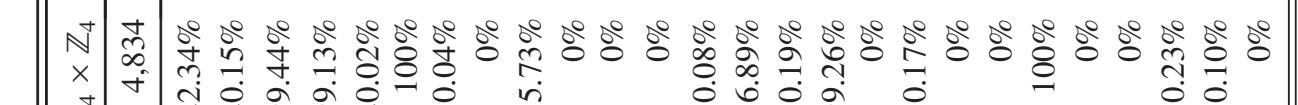

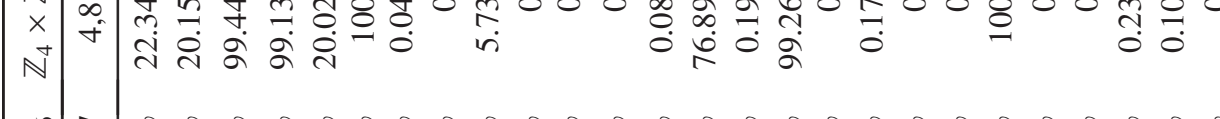

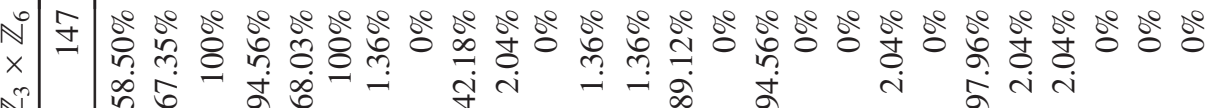

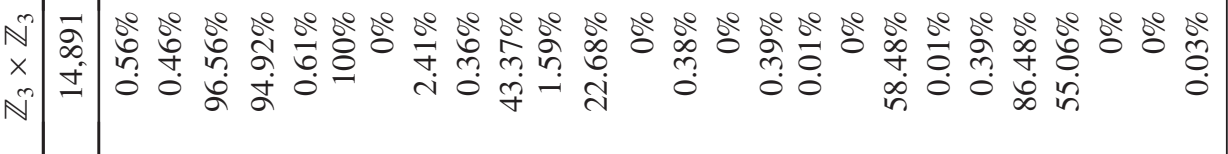

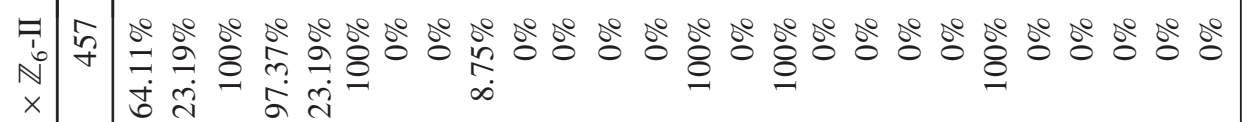

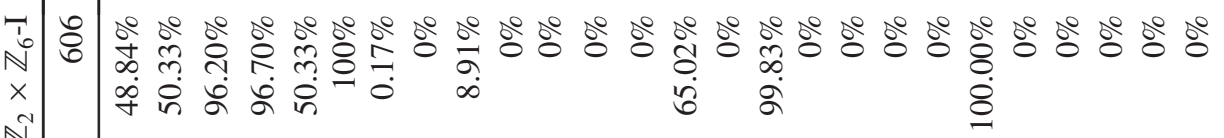

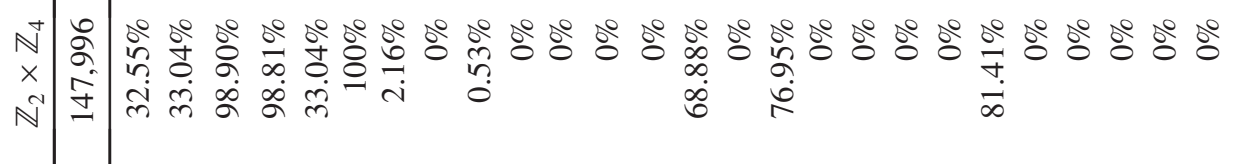

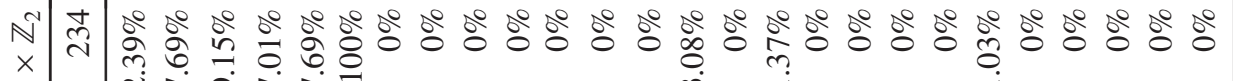
N तु

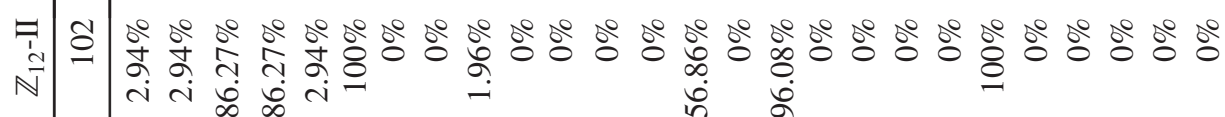

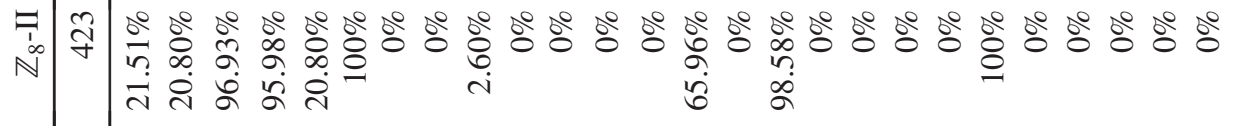

雨

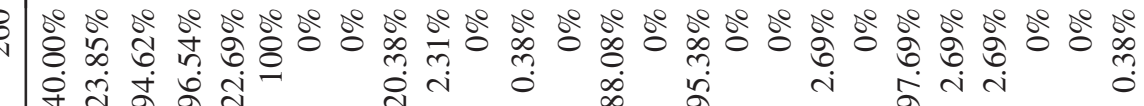

虫

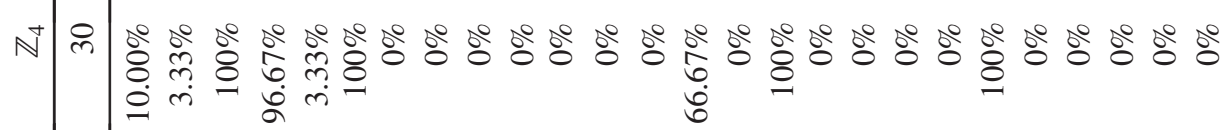

约 


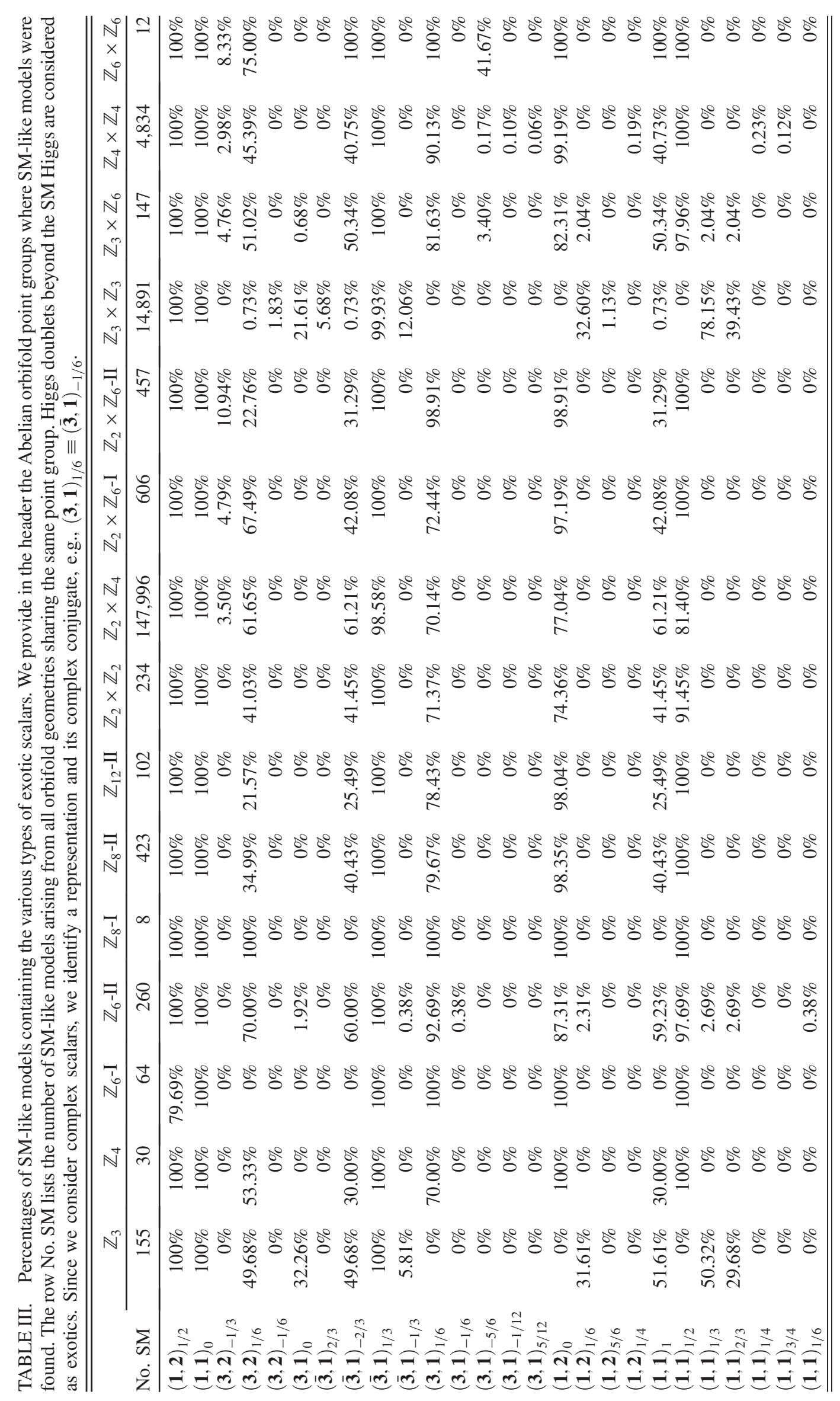




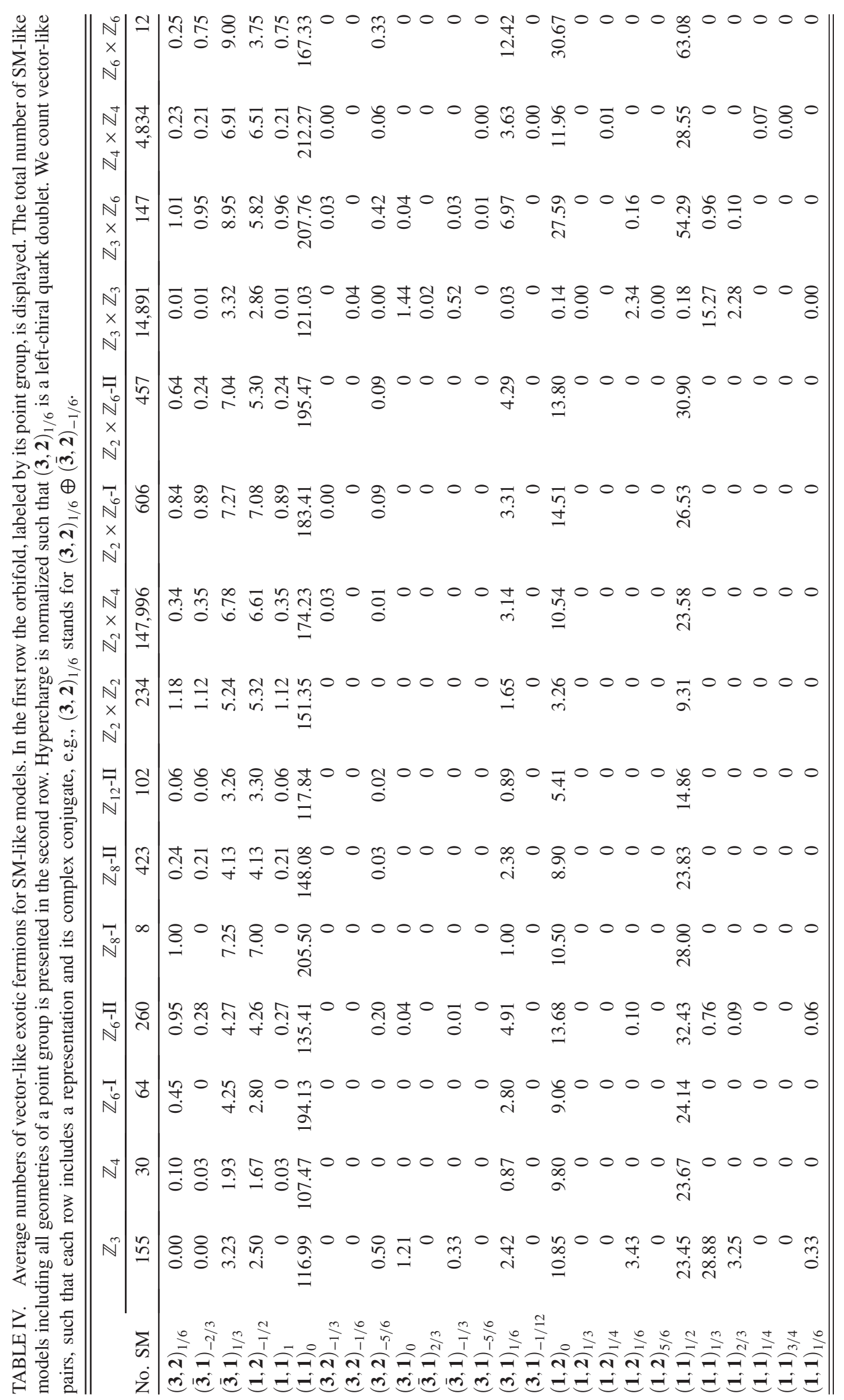




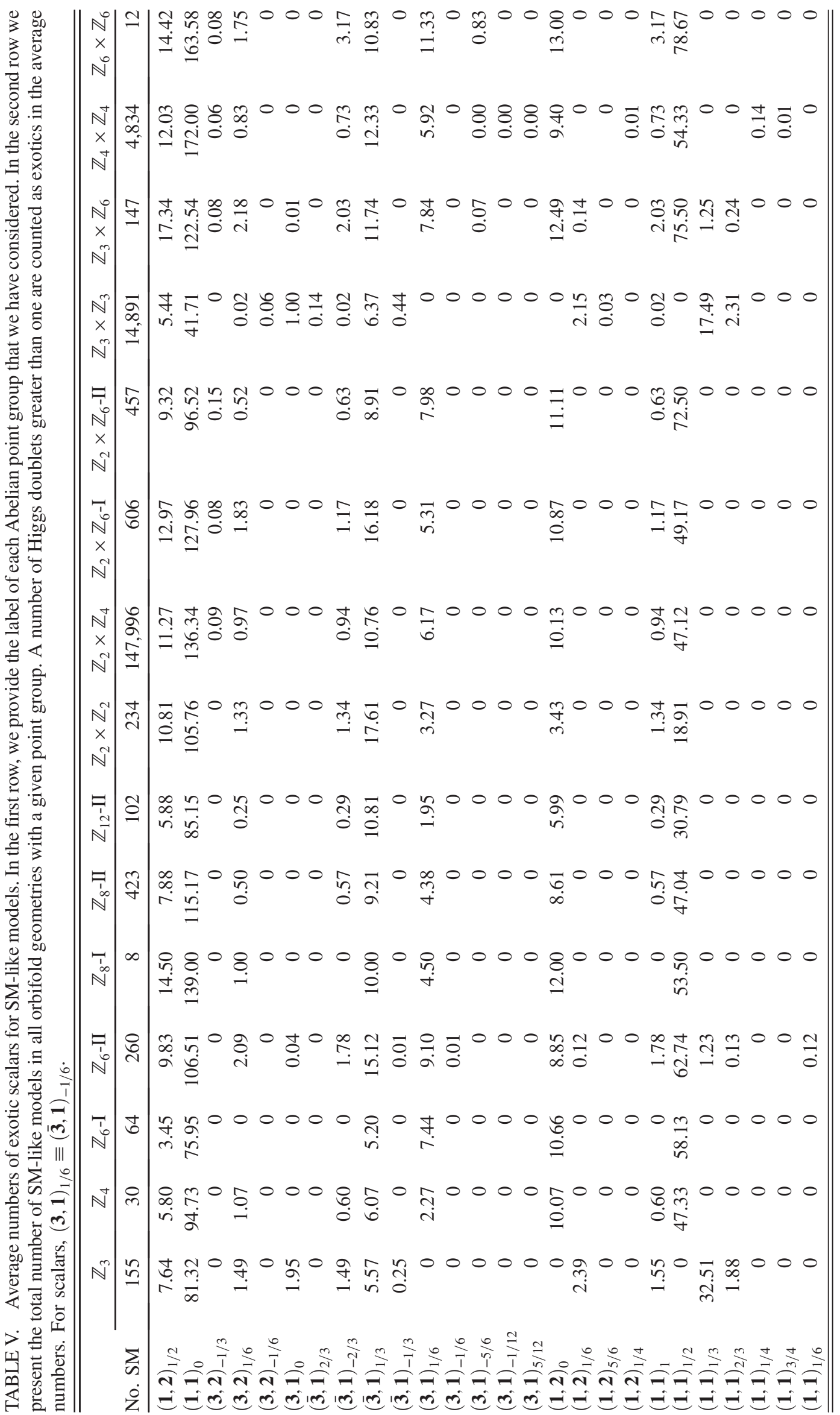




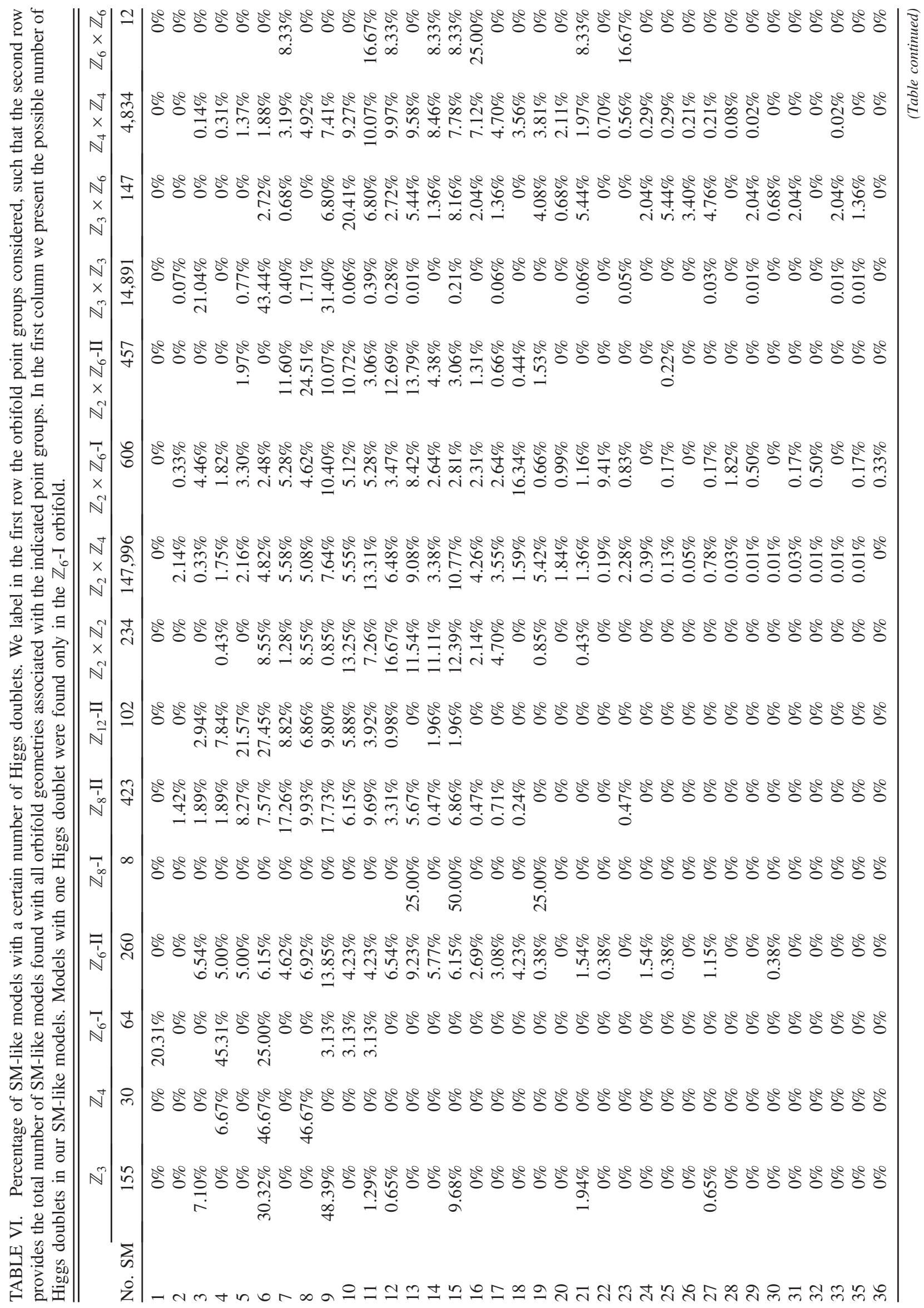




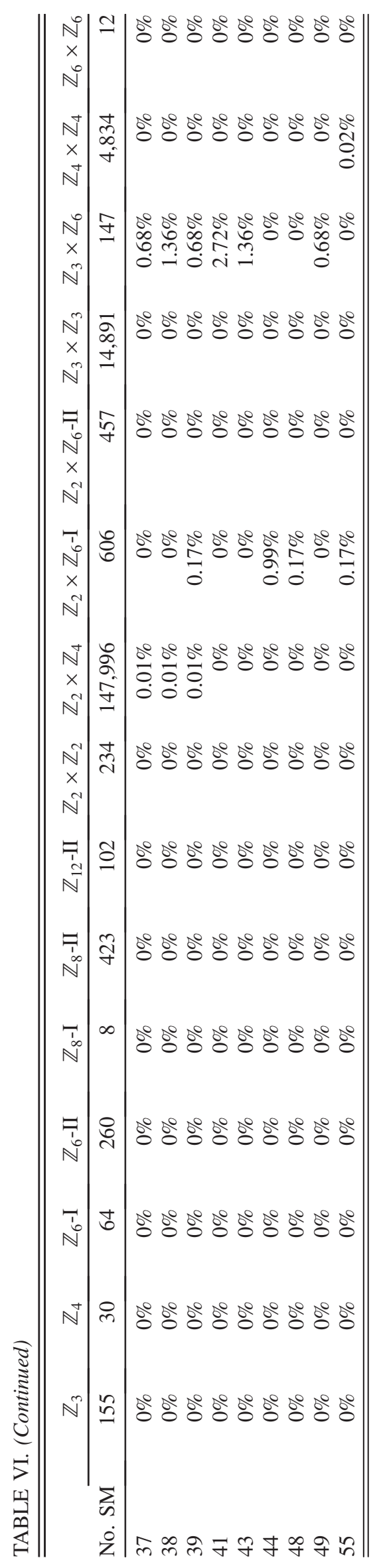




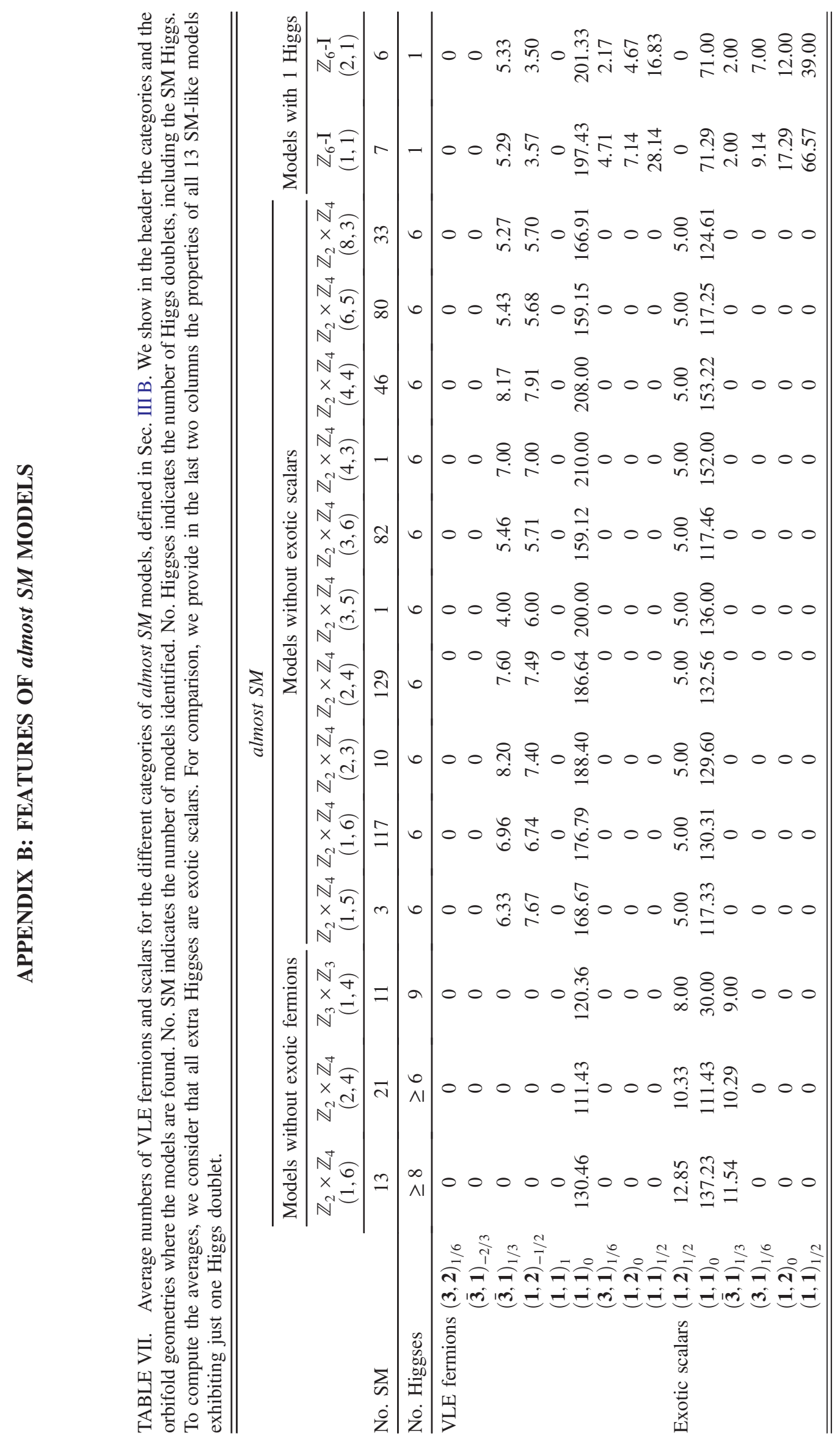


TABLE VIII. Number of almost SM orbifold models without exotic fermions (see Table VII) with various numbers of Higgs doublets, including the SM Higgs.

\begin{tabular}{|c|c|c|c|c|c|}
\hline \multirow{2}{*}{$\begin{array}{l}\text { Orbifold } \\
\text { Geometry }\end{array}$} & \multicolumn{5}{|c|}{ No. of almost SM models with $n$ Higgs doublets } \\
\hline & $n=6$ & 8 & 10 & 14 & 18 \\
\hline $\mathbb{Z}_{2} \times \mathbb{Z}_{4}(1,6)$ & 0 & 1 & 3 & 5 & 4 \\
\hline $\mathbb{Z}_{2} \times \mathbb{Z}_{4}(2,4)$ & 3 & 0 & 9 & 8 & 1 \\
\hline
\end{tabular}

TABLE IX. Massless spectra of three promising SM-like models. The representations are written with respect to $\mathcal{G}_{\mathrm{SM}}=\mathrm{SU}(3)_{C} \times \mathrm{SU}(2)_{L} \times \mathrm{U}(1)_{Y}$, where the hypercharges are displayed as subscripts. All fermions are left-handed. Model 1 is an almost $S M$ based on the $\mathbb{Z}_{2} \times \mathbb{Z}_{4}(2,4)$ orbifold geometry, model 2 is an almost $S M$ based on the $\mathbb{Z}_{3} \times \mathbb{Z}_{3}(1,4)$ orbifold geometry, and model 3 is an SM-like string model arising from the $\mathbb{Z}_{2} \times \mathbb{Z}_{2}(12,1)$ geometry. Besides the numbers of Higgs doublets $\phi$, right-handed neutrinos $\bar{\nu}$, and flavons $s$, the models differ by the extra "hidden" non-Abelian gauge factor which acts as a continuous gauge flavor symmetry.

\begin{tabular}{lcc}
\hline \hline & Model 1 & \\
\hline No. & Fermionic irrep & Label \\
\hline 5 & $(\mathbf{1}, \mathbf{2})_{-1 / 2}$ & $\ell_{i}$ \\
2 & $(\mathbf{1}, \mathbf{2})_{1 / 2}$ & $\bar{\ell}_{i}$ \\
3 & $(\mathbf{1}, \mathbf{1})_{1}$ & $\bar{e}_{i}$ \\
3 & $(\mathbf{3}, \mathbf{2})_{1 / 6}$ & $q_{i}$ \\
3 & $(\overline{\mathbf{3}}, \mathbf{1})_{-2 / 3}$ & $\bar{u}_{i}$ \\
7 & $(\overline{\mathbf{3}}, \mathbf{1})_{1 / 3}$ & $\bar{d}_{i}$ \\
4 & $(\mathbf{3}, \mathbf{1})_{-1 / 3}$ & $d_{i}$ \\
111 & $(\mathbf{1}, \mathbf{1})_{0}$ & $\bar{\nu}_{i}$ \\
\hline
\end{tabular}

TABLE IX. (Continued)

\begin{tabular}{lcc}
\hline \hline & Model 1 & \\
\hline No. & Scalar irrep & Label \\
6 & $(\mathbf{1}, \mathbf{2})_{1 / 2}$ & $\phi_{i}$ \\
76 & $(\mathbf{1}, \mathbf{1})_{0}$ & $s_{i}$ \\
\hline \hline & Model 2 & \\
\hline No. & Fermionic irrep & Label \\
\hline 3 & $(\mathbf{1}, \mathbf{2})_{-1 / 2}$ & $\ell_{i}$ \\
3 & $(\mathbf{1}, \mathbf{1})_{1}$ & $\bar{e}_{i}$ \\
3 & $(\mathbf{3}, \mathbf{2})_{1 / 6}$ & $q_{i}$ \\
3 & $(\overline{\mathbf{3}}, \mathbf{1})_{-2 / 3}$ & $\bar{u}_{i}$ \\
3 & $(\overline{\mathbf{3}}, \mathbf{1})_{1 / 3}$ & $\bar{d}_{i}$ \\
119 & $(\mathbf{1}, \mathbf{1})_{0}$ & $\bar{\nu}_{i}$ \\
No. & Scalar irrep & Label \\
9 & $(\mathbf{1}, \mathbf{2})_{1 / 2}$ & $\phi_{i}$ \\
30 & $(\mathbf{1}, \mathbf{1})_{0}$ & $s_{i}$ \\
9 & $(\overline{\mathbf{3}}, \mathbf{1})_{1 / 3}$ & $S_{1, i}$ \\
& & \\
\hline \hline
\end{tabular}

Model 3

\begin{tabular}{lcc}
\hline No. & Fermionic irrep & Label \\
\hline 3 & $(\mathbf{1}, \mathbf{2})_{-1 / 2}$ & $\ell_{i}$ \\
3 & $(\mathbf{1}, \mathbf{1})_{-1}$ & $e_{i}$ \\
6 & $(\mathbf{1}, \mathbf{1})_{1}$ & $\bar{e}_{i}$ \\
3 & $(\mathbf{3}, \mathbf{2})_{1 / 6}$ & $q_{i}$ \\
6 & $(\overline{\mathbf{3}}, \mathbf{1})_{-2 / 3}$ & $\bar{u}_{i}$ \\
3 & $(\mathbf{3}, \mathbf{1})_{2 / 3}$ & $u_{i}$ \\
5 & $(\overline{\mathbf{3}}, \mathbf{1})_{1 / 3}$ & $\bar{d}_{i}$ \\
2 & $(\mathbf{3}, \mathbf{1})_{-1 / 3}$ & $d_{i}$ \\
67 & $(\mathbf{1}, \mathbf{1})_{0}$ & $\bar{\nu}_{i}$ \\
No. & Scalar irrep & Label \\
6 & $(\mathbf{1}, \mathbf{2})_{1 / 2}$ & $\phi_{i}$ \\
24 & $(\mathbf{1}, \mathbf{1})_{0}$ & $s_{i}$ \\
12 & $(\overline{\mathbf{3}}, \mathbf{1})_{1 / 3}$ & $S_{1, i}$ \\
\hline \hline
\end{tabular}




\section{APPENDIX C: CORRELATIONS IN THE LANDSCAPE OF SM-LIKE STRING MODELS}
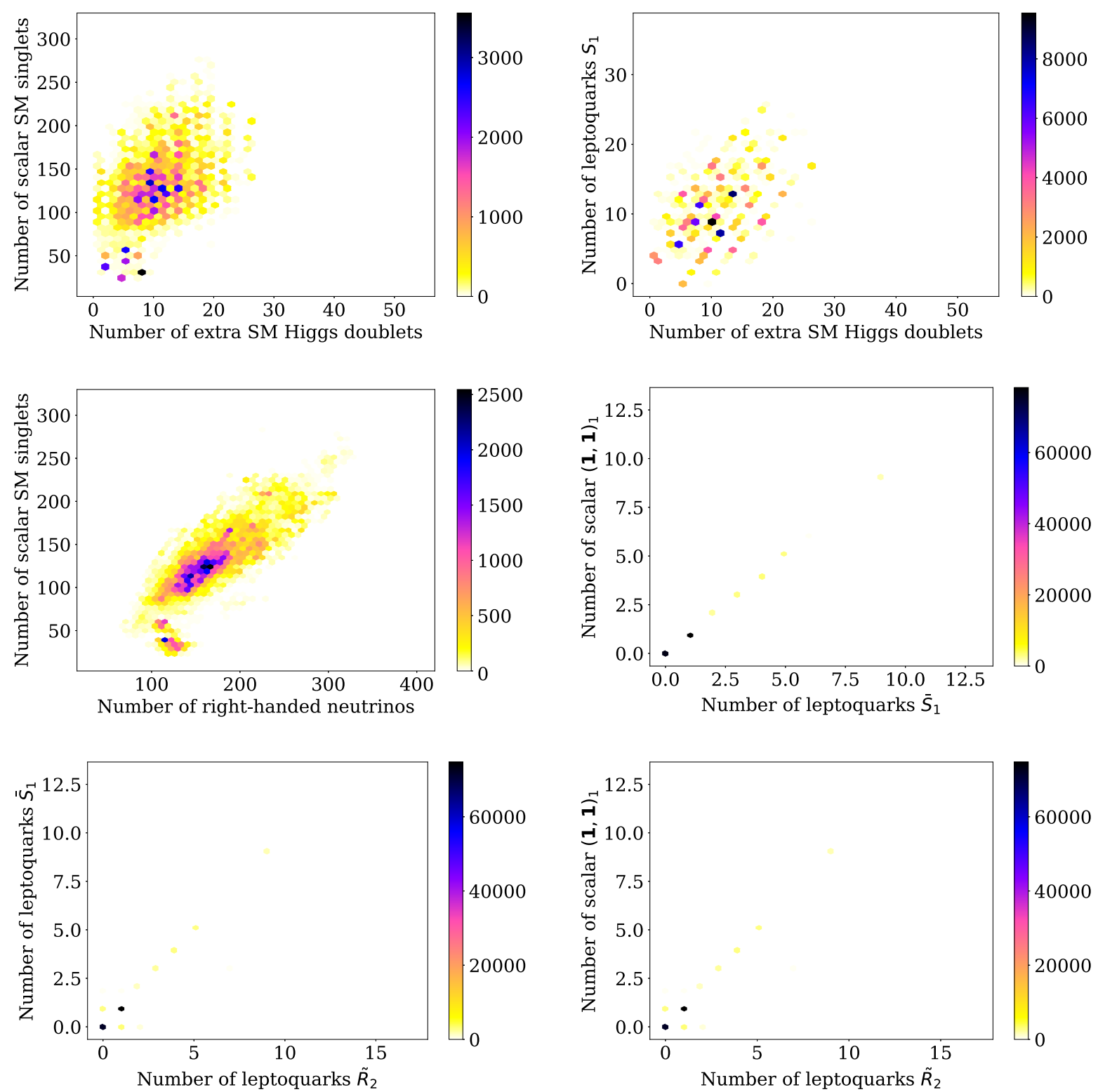

FIG. 5. Some (high) correlations between numbers of exotics in our data set of 170219 SM-like string models. The correlations are computed on the full data set and they read from left to right and top to bottom: $0.49,0.49,0.81,0.99,0.91$ and 0.91 . They point towards the existence of local GUTs [12,97-99], where at certain orbifold singularities complete GUT representations are localized even though the four-dimensional gauge group is just $\mathcal{G}_{\mathrm{SM}}$. The correlation between the number of extra Higgs doublets and the number of scalar color triplets $S_{1}$ seems to originate from complete scalar 5-plets of local SU(5) GUTs, while the correlations between the number of Higgs doublets and the scalar leptoquarks $\bar{S}_{1}$ and $\tilde{R}_{2}$ might result from complete scalar 10-plets of SU(5). 
[1] L. J. Dixon, J. A. Harvey, C. Vafa, and E. Witten, Strings on orbifolds, Nucl. Phys. B261, 678 (1985).

[2] L. J. Dixon, J. A. Harvey, C. Vafa, and E. Witten, Strings on orbifolds. 2., Nucl. Phys. B274, 285 (1986).

[3] L. E. Ibáñez, H. P. Nilles, and F. Quevedo, Orbifolds and Wilson lines, Phys. Lett. B 187, 25 (1987).

[4] L. E. Ibáñez, J. E. Kim, H. P. Nilles, and F. Quevedo, Orbifold compactifications with three families of $\mathrm{SU}(3) \times \mathrm{SU}(2) \times \mathrm{U}(1)$ n, Phys. Lett. B 191, 282 (1987).

[5] S. Förste, T. Kobayashi, H. Ohki, and K.-J. Takahashi, Non-Factorisable $\mathrm{Z}(2) \times \mathrm{Z}(2)$ Heterotic Orbifold Models and Yukawa Couplings, J. High Energy Phys. 03 (2007) 011.

[6] T. Kobayashi, S. Raby, and R.-J. Zhang, Searching for realistic 4d string models with a Pati-Salam symmetry: Orbifold grand unified theories from heterotic string compactification on a Z(6) orbifold, Nucl. Phys. B704, 3 (2005).

[7] W. Buchmüller, K. Hamaguchi, O. Lebedev, and M. Ratz, Supersymmetric Standard Model from the Heterotic String, Phys. Rev. Lett. 96, 121602 (2006).

[8] O. Lebedev, H. P. Nilles, S. Raby, S. Ramos-Sánchez, M. Ratz, P. K. S. Vaudrevange, and A. Wingerter, A MiniLandscape of exact MSSM spectra in heterotic orbifolds, Phys. Lett. B 645, 88 (2007).

[9] J. E. Kim, J.-H. Kim, and B. Kyae, Superstring standard model from Z(12-I) orbifold compactification with and without exotics, and effective R-parity, J. High Energy Phys. 06 (2007) 034

[10] O. Lebedev, H. P. Nilles, S. Ramos-Sánchez, M. Ratz, and P. K. S. Vaudrevange, Heterotic mini-landscape (II): Completing the search for MSSM vacua in a $Z_{6}$ orbifold, Phys. Lett. B 668, 331 (2008).

[11] D. K. M. Peña, H. P. Nilles, and P.-K. Oehlmann, A Zipcode for Quarks, Leptons and Higgs Bosons, J. High Energy Phys. 12 (2012) 024.

[12] H. P. Nilles and P. K. S. Vaudrevange, Geography of fields in extra dimensions: String theory lessons for particle physics, Mod. Phys. Lett. A 30, 1530008 (2015).

[13] P. Athanasopoulos, A. E. Faraggi, S. G. Nibbelink, and V. M. Mehta, Heterotic free fermionic and symmetric toroidal orbifold models, J. High Energy Phys. 04 (2016) 038.

[14] Y. Olguín-Trejo, R. Pérez-Martínez, and S. Ramos-Sánchez, Charting the flavor landscape of MSSM-like Abelian heterotic orbifolds, Phys. Rev. D 98, 106020 (2018).

[15] E. Parr and P. K. S. Vaudrevange, Contrast data mining for the MSSM from strings, Nucl. Phys. B952, 114922 (2020).

[16] E. Parr, P. K. S. Vaudrevange, and M. Wimmer, Predicting the orbifold origin of the MSSM, Fortschr. Phys. 68, 2000032 (2020).

[17] P. Ko, T. Kobayashi, and J.-H. Park, Lepton masses and mixing angles from heterotic orbifold models, Phys. Rev. D 71, 095010 (2005).

[18] T. Araki, K.-S. Choi, T. Kobayashi, J. Kubo, and H. Ohki, Discrete R-symmetry anomalies in heterotic orbifold models, Phys. Rev. D 76, 066006 (2007).

[19] O. Lebedev, H. P. Nilles, S. Raby, S. Ramos-Sánchez, M. Ratz, P. K. S. Vaudrevange, and A. Wingerter, The heterotic road to the MSSM with R parity, Phys. Rev. D 77, 046013 (2008).
[20] W. Buchmüller, K. Hamaguchi, O. Lebedev, S. Ramos-Sánchez, and M. Ratz, Seesaw Neutrinos from the Heterotic String, Phys. Rev. Lett. 99, 021601 (2007).

[21] O. Lebedev and S. Ramos-Sánchez, The NMSSM and string theory, Phys. Lett. B 684, 48 (2010).

[22] J. Ashfaque, L. Delle Rose, A. E. Faraggi, and C. Marzo, LHC di-photon excess and gauge coupling unification in extra $Z^{\prime}$ heterotic-string derived models, Eur. Phys. J. C 76, 570 (2016).

[23] B. Carballo-Pérez, E. Peinado, and S. Ramos-Sánchez, $\Delta(54)$ flavor phenomenology and strings, J. High Energy Phys. 12 (2016) 131.

[24] Y. Olguín-Trejo, O. Pérez-Figueroa, R. Pérez-Martínez, and S. Ramos-Sánchez, $\mathrm{U}(1)^{\prime}$ coupling constant at low energies from heterotic orbifolds, Phys. Lett. B 795, 673 (2019).

[25] J. Jeong, J. E. Kim, and S. Nam, Leptonic $C P$ violation in flipped SU(5) GUT from $Z_{12}$-I orbifold compactification, Phys. Lett. B 791, 215 (2019).

[26] J. E. Kim, R-parity from string compactification, Phys. Rev. D 104, 016012 (2021).

[27] D. J. Gross, J. A. Harvey, E. J. Martinec, and R. Rohm, The Heterotic String, Phys. Rev. Lett. 54, 502 (1985).

[28] L. J. Dixon and J. A. Harvey, String theories in tendimensions without space-time supersymmetry, Nucl. Phys. B274, 93 (1986).

[29] L. Álvarez-Gaumé, P. H. Ginsparg, G. W. Moore, and C. Vafa, An $\mathrm{O}(16) \times \mathrm{O}(16)$ heterotic string, Phys. Lett. B 171, 155 (1986).

[30] M. Fischer, M. Ratz, J. Torrado, and P. K. S. Vaudrevange, Classification of symmetric toroidal orbifolds, J. High Energy Phys. 01 (2013) 084.

[31] M. Blaszczyk, S. G. Nibbelink, O. Loukas, and S. RamosSánchez, Non-supersymmetric heterotic model building, J. High Energy Phys. 10 (2014) 119.

[32] J. M. Ashfaque, P. Athanasopoulos, A. E. Faraggi, and H. Sonmez, Non-tachyonic semi-realistic non-supersymmetric heterotic string vacua, Eur. Phys. J. C 76, 208 (2016).

[33] A. E. Faraggi, V. G. Matyas, and B. Percival, Type $0 \mathbb{Z}_{2} \times$ $\mathbb{Z}_{2}$ heterotic string orbifolds and misaligned supersymmetry, arXiv:2010.06637.

[34] A. E. Faraggi, V. G. Matyas, and B. Percival, Classification of nonsupersymmetric Pati-Salam heterotic string models, Phys. Rev. D 104, 046002 (2021).

[35] A. E. Faraggi, V. G. Matyas, and B. Percival, Type $\overline{0}$ heterotic string orbifolds, Phys. Lett. B 814, 136080 (2021).

[36] M. Blaszczyk, S. G. Nibbelink, O. Loukas, and F. Ruehle, Calabi-Yau compactifications of non-supersymmetric heterotic string theory, J. High Energy Phys. 10 (2015) 166.

[37] S. Abel, K. R. Dienes, and E. Mavroudi, Towards a nonsupersymmetric string phenomenology, Phys. Rev. D 91, 126014 (2015).

[38] S. Abel, K. R. Dienes, and E. Mavroudi, GUT precursors and entwined SUSY: The phenomenology of stable nonsupersymmetric strings, Phys. Rev. D 97, 126017 (2018).

[39] C. Angelantonj, I. Florakis, and M. Tsulaia, Universality of gauge thresholds in non-supersymmetric heterotic vacua, Phys. Lett. B 736, 365 (2014). 
[40] I. Florakis and J. Rizos, Chiral heterotic strings with positive cosmological constant, Nucl. Phys. B913, 495 (2016).

[41] I. Florakis and J. Rizos, A solution to the decompactification problem in chiral heterotic strings, Nucl. Phys. B921, 1 (2017).

[42] H. P. Nilles, S. Ramos-Sánchez, P. K. S. Vaudrevange, and A. Wingerter, The orbifolder: A tool to study the low energy effective theory of heterotic orbifolds, Comput. Phys. Commun. 183, 1363 (2012).

[43] W. Buchmüller, R. Rückl, and D. Wyler, Leptoquarks in lepton—quark collisions, Phys. Lett. B 191, 442 (1987); 448, 320(E) (1999).

[44] I. Doršner, S. Fajfer, A. Greljo, J. F. Kamenik, and N. Košnik, Physics of leptoquarks in precision experiments and at particle colliders, Phys. Rep. 641, 1 (2016).

[45] B. Diaz, M. Schmaltz, and Y.-M. Zhong, The leptoquark Hunter's guide: Pair production, J. High Energy Phys. 10 (2017) 097.

[46] P. Zyla et al. (Particle Data Group), Review of particle physics, Prog. Theor. Exp. Phys. 2020, 083C01 (2020).

[47] L. A. Anchordoqui, I. Antoniadis, X. Huang, D. Lüst, and T. R. Taylor, Muon $g-2$ discrepancy within D-brane string compactifications, Fortschr. Phys. 2100084 (2021).

[48] L. A. Anchordoqui, I. Antoniadis, X. Huang, D. Lust, and T. R. Taylor, Leptophilic U(1) massive vector bosons from large extra dimensions, arXiv:2105.02630.

[49] H. M. Lee, Leptoquark option for $B$-meson anomalies and leptonic signatures, Phys. Rev. D 104, 015007 (2021).

[50] P. Fileviez Pérez, C. Murgui, and A. D. Plascencia, Leptoquarks and matter unification: Flavor anomalies and the muon $g-2$, arXiv:2104.11229.

[51] A. Angelescu, D. Bečirević, D. A. Faroughy, F. Jaffredo, and O. Sumensari, On the single leptoquark solutions to the $B$-physics anomalies, arXiv:2103.12504.

[52] I. Doršner, S. Fajfer, and O. Sumensari, Muon $g-2$ and scalar leptoquark mixing, J. High Energy Phys. 06 (2020) 089.

[53] P. Athron, C. Balázs, D. H. Jacob, W. Kotlarski, D. Stöckinger, and H. Stöckinger-Kim, New physics explanations of $a_{\mu}$ in light of the FNAL muon $g-2$ measurement, arXiv:2104.03691.

[54] D. Marzocca and S. Trifinopoulos, Minimal Explanation of Flavor Anomalies: B -Meson Decays, Muon Magnetic Moment, and the Cabibbo Angle, Phys. Rev. Lett. 127, 061803 (2021).

[55] T. Nomura and H. Okada, Explanations for anomalies of muon anomalous magnetic dipole moment, $b \rightarrow s \mu \bar{\mu}$ and radiative neutrino masses in a leptoquark model, arXiv:2104.03248.

[56] W.-Y. Keung, D. Marfatia, and P.-Y. Tseng, Axion-like particles, two-Higgs-doublet models, leptoquarks, and the electron and muon $g-2$, Lett. High Energy Phys. 2021, 209 (2021).

[57] W. Yin, Radiative lepton mass and muon $g-2$ with suppressed lepton flavor and CP violations, arXiv:2103 .14234.

[58] Y. Cai, J. Gargalionis, M. A. Schmidt, and R. R. Volkas, Reconsidering the One Leptoquark solution: Flavor anomalies and neutrino mass, J. High Energy Phys. 10 (2017) 047.
[59] S.-M. Choi, Y.-J. Kang, H. M. Lee, and T.-G. Ro, Leptoquark portal dark matter, J. High Energy Phys. 10 (2018) 104.

[60] I. Dorsner, S. Fajfer, and N. Kosnik, Heavy and light scalar leptoquarks in proton decay, Phys. Rev. D 86, 015013 (2012).

[61] Y.-H. He, Deep-learning the landscape, arXiv:1706.02714.

[62] F. Ruehle, Evolving neural networks with genetic algorithms to study the String Landscape, J. High Energy Phys. 08 (2017) 038.

[63] J. Carifio, J. Halverson, D. Krioukov, and B. D. Nelson, Machine learning in the string landscape, J. High Energy Phys. 09 (2017) 157.

[64] J. Carifio, W. J. Cunningham, J. Halverson, D. Krioukov, C. Long, and B.D. Nelson, Vacuum Selection from Cosmology on Networks of String Geometries, Phys. Rev. Lett. 121, 101602 (2018).

[65] A. Mütter, E. Parr, and P. K. S. Vaudrevange, Deep learning in the heterotic orbifold landscape, Nucl. Phys. B940, 113 (2019).

[66] J. Halverson, B. Nelson, and F. Ruehle, Branes with brains: Exploring string vacua with deep reinforcement learning, J. High Energy Phys. 06 (2019) 003.

[67] A. Cole, A. Schachner, and G. Shiu, Searching the landscape of flux vacua with genetic algorithms, J. High Energy Phys. 11 (2019) 045.

[68] J. Halverson and C. Long, Statistical predictions in string theory and deep generative models, Fortschr. Phys. 68, 2000005 (2020).

[69] H. Otsuka and K. Takemoto, Deep learning and k-means clustering in heterotic string vacua with line bundles, J. High Energy Phys. 05 (2020) 047.

[70] M. Larfors and R. Schneider, Explore and exploit with heterotic line bundle models, Fortschr. Phys. 68, 2000034 (2020).

[71] R. Deen, Y.-H. He, S.-J. Lee, and A. Lukas, Machine learning string standard models, arXiv:2003.13339.

[72] L. B. Anderson, M. Gerdes, J. Gray, S. Krippendorf, N. Raghuram, and F. Ruehle, Moduli-dependent Calabi-Yau and $S U(3)$-structure metrics from machine learning, J. High Energy Phys. 05 (2021) 013.

[73] F. Ruehle, Data science applications to string theory, Phys. Rep. 839, 1 (2020).

[74] N. C. Bizet, C. Damian, O. Loaiza-Brito, D. K. M. Peña, and J.M. Barrera, Testing swampland conjectures with machine learning, Eur. Phys. J. C 80, 766 (2020).

[75] I. Bena, J. Blåbäck, M. Graña, and S. Lüst, Algorithmically solving the tadpole problem, arXiv:2103.03250.

[76] S. Ramos-Sánchez, Towards low energy physics from the heterotic string, Fortschr. Phys. 57, 907 (2009).

[77] P. K. S. Vaudrevange, Grand unification in the heterotic brane world, arXiv:0812.3503.

[78] F. Plöger, S. Ramos-Sánchez, M. Ratz, and P. K. S. Vaudrevange, Mirage torsion, J. High Energy Phys. 04 (2007) 063.

[79] M. B. Green and J. H. Schwarz, Anomaly cancellation in supersymmetric $D=10$ gauge theory and superstring theory, Phys. Lett. 149B, 117 (1984).

[80] D. Bečirević, I. Doršner, S. Fajfer, N. Košnik, D. A. Faroughy, and O. Sumensari, Scalar leptoquarks from 
grand unified theories to accommodate the $B$-physics anomalies, Phys. Rev. D 98, 055003 (2018).

[81] J. A. Arroyo and S. Ramos-Sánchez, One-loop corrections for Higgs-portal dark matter, J. Phys. Conf. Ser. 761, 012014 (2016).

[82] G. Arcadi, A. Djouadi, and M. Raidal, Dark matter through the Higgs portal, Phys. Rep. 842, 1 (2020).

[83] C. D. Froggatt and H. B. Nielsen, Hierarchy of quark masses, cabibbo angles and $C P$ violation, Nucl. Phys. B147, 277 (1979).

[84] K. S. Babu, Topics in flavor physics, in The Dawn of the LHC Era (World Scientific Publishing, Singapore, 2009), https://doi.org/10.1142/9789812838360_0002.

[85] A. N. Schellekens, Electric charge quantization in string theory, Phys. Lett. B 237, 363 (1990).

[86] A. Barroso, P. M. Ferreira, R. Santos, and J. P. Silva, Stability of the normal vacuum in multi-Higgs-doublet models, Phys. Rev. D 74, 085016 (2006).

[87] P. M. Ferreira and J. P. Silva, Discrete and continuous symmetries in multi-Higgs-doublet models, Phys. Rev. D 78, 116007 (2008).

[88] I. P. Ivanov, V. Keus, and E. Vdovin, Abelian symmetries in multi-Higgs-doublet models, J. Phys. A 45, 215201 (2012).

[89] C.-F. Chang, X.-G. He, and J. Tandean, Two-Higgsdoublet-portal dark-matter models in light of direct search and LHC data, J. High Energy Phys. 04 (2017) 107.

[90] V. Keus, S. F. King, and S. Moretti, Three-Higgs-doublet models: Symmetries, potentials and Higgs boson masses, J. High Energy Phys. 01 (2014) 052.

[91] M. Alakhras, N. Chamoun, X.-L. Chen, C.-S. Huang, and C. Liu, Six Higgs doublets model for Dark Matter, Phys. Rev. D 96, 095013 (2017).

[92] X.-F. Han, T. Li, H.-X. Wang, L. Wang, and Y. Zhang, Lepton-specific inert two-Higgs-doublet model confronted with the new results for muon and electron g-2 anomalies and multi-lepton searches at the LHC, arXiv:2104.03227.

[93] C.-H. Chen, C.-W. Chiang, and T. Nomura, Muon $g-2$ in two-Higgs-doublet model with type-II seesaw mechanism, arXiv:2104.03275.

[94] P. M. Ferreira, B. L. Gonçalves, F. R. Joaquim, and M. Sher, $(g-2)_{\mu}$ in the 2HDM and slightly beyond-an updated view, arXiv:2104.03367.

[95] A. E. Cárcamo Hernández, S. Kovalenko, M. Maniatis, and I. Schmidt, Fermion mass hierarchy and g-2 anomalies in an extended 3HDM Model, arXiv:2104.07047.

[96] A. Jueid, J. Kim, S. Lee, and J. Song, Type-X two Higgs doublet model in light of the muon $g-2$ : confronting Higgs and collider data, arXiv:2104.10175.
[97] S. Förste, H. P. Nilles, P. K. S. Vaudrevange, and A. Wingerter, Heterotic brane world, Phys. Rev. D 70, 106008 (2004).

[98] W. Buchmüller, K. Hamaguchi, O. Lebedev, and M. Ratz, Dual models of gauge unification in various dimensions, Nucl. Phys. B712, 139 (2005).

[99] M. Ratz, Notes on local grand unification, Soryushiron Kenkyu Electron. 116, A56 (2008).

[100] R. Pérez-Martínez, S. Ramos-Sánchez, and P. K. Vaudrevange, Non-supersymmetric orbifolds: Model definitions and spectra, 2021, http://stringpheno.fisica.unam.mx/ nonsusy-orbifolds/.

[101] A. Greljo, P. Stangl, and A. E. Thomsen, A model of muon anomalies, arXiv:2103.13991.

[102] H. P. Nilles, S. Ramos-Sánchez, and P. K. S. Vaudrevange, Lessons from eclectic flavor symmetries, Nucl. Phys. B957, 115098 (2020).

[103] H. P. Nilles, S. Ramos-Sánchez, and P. K. S. Vaudrevange, Eclectic flavor scheme from ten-dimensional string theory-I. Basic results, Phys. Lett. B 808, 135615 (2020).

[104] A. Baur, M. Kade, H. P. Nilles, S. Ramos-Sánchez, and P. K. S. Vaudrevange, The eclectic flavor symmetry of the $\mathbb{Z}_{2}$ orbifold, J. High Energy Phys. 02 (2021) 018 .

[105] T. Kobayashi, H. P. Nilles, F. Plöger, S. Raby, and M. Ratz, Stringy origin of non-Abelian discrete flavor symmetries, Nucl. Phys. B768, 135 (2007).

[106] Y. Satoh, Y. Sugawara, and T. Wada, Non-supersymmetric asymmetric orbifolds with vanishing cosmological constant, J. High Energy Phys. 02 (2016) 184.

[107] S. G. Nibbelink, O. Loukas, A. Mütter, E. Parr, and P. K. S. Vaudrevange, Tension between a vanishing cosmological constant and non-supersymmetric heterotic orbifolds, Fortschr. Phys. 68, 2000044 (2020).

[108] I. Basile, J. Mourad, and A. Sagnotti, On classical stability with broken supersymmetry, J. High Energy Phys. 01 (2019) 174.

[109] R. Antonelli and I. Basile, Brane annihilation in nonsupersymmetric strings, J. High Energy Phys. 11 (2019) 021.

[110] K. Aoyama and Y. Sugawara, Non-SUSY Gepner models with vanishing cosmological constant, Prog. Theor. Exp. Phys. 2020, 103B01 (2020).

[111] K. Aoyama and Y. Sugawara, Non-SUSY heterotic string vacua of Gepner models with vanishing cosmological constant, Prog. Theor. Exp. Phys. 2021, 033 B03 (2021). 